\title{
Detailed Passive Cable Models of Whole-Cell Recorded CA3 Pyramidal Neurons in Rat Hippocampal Slices
}

\author{
Guy Major,' Alan U. Larkman,, Peter Jonas, $^{2}$ Bert Sakmann, ${ }^{2}$ and J. Julian B. Jack' \\ 'University Laboratory of Physiology, Oxford, OX1 3PT, United Kingdom and ${ }^{2}$ Max-Planck-Institut für Medizinische \\ Forschung, Abteilung Zellphysiologie, D-69120 Heidelberg, Germany
}

Tight-seal whole-cell recordings were made from cleaned somata of CA3 pyramidal cells deep in hippocampal slices from 19-21-d-old rats. The cells were filled with biocytin, and their voltage responses to short current pulses were recorded. After washout of initial sag, responses scaled linearly with injected current and were stable over time. The dendritic and axonal arbors of four cells were reconstructed and measured using light microscopy. Dendritic spines and axonal boutons were counted and the additional membrane area was incorporated into the relevant segments. The morphology of each neuron was converted into a detailed branching cable model by assuming values for specific membrane capacitance $C_{m}$ and resistance $R_{m}$, and cytoplasmic resistivity $\boldsymbol{R}_{i}$. These parameters were optimized for each cell by directly matching the model's response to that of the real cell by means of a modified weighted least-squares fitting procedure. By comparing the deviations between model and experimental responses to control noise recordings, approximate $95 \%$ confidence intervals were established for each parameter. If a somatic shunt was allowed, a wide range of possible $\boldsymbol{R}_{m}$ values produced acceptable fits. With zero shunt, $C_{m}$ was $0.7-0.8 \mu \mathrm{Fcm}^{-2}, R_{i}$ was $170-340 \Omega \mathrm{cm}$, and $R_{m}$ ranged between 120 and $200 \mathrm{k} \Omega \mathrm{cm}^{2}$. The electrotonic lengths of the basal and oblique dendrites were $0.2-0.3$ space constants, and those of the apical tufts were 0.4-0.7 space constants. The steady-state electrical geometry of these cells was therefore compact; average dendritic tip/soma relative synaptic efficacies were $>\mathbf{9 3} \%$ for the basal and oblique dendrites, and $>\mathbf{8 1} \%$ for the tufts. With fast transient synaptic inputs, however, the models produced a wide range of postsynaptic potential shapes and marked filtering of voltage-clamp currents.

[Key words: passive cable model, CA3 pyramidal cell, whole-cell recording, biocytin, direct fit, morphological reconstruction, electrical parameters, voltage clamp, cytoplasmic resistivity, specific membrane capacitance]

\footnotetext{
Received Oct. 11, 1993; revised Jan. 19, 1994; accepted Jan. 27, 1994.

G.M. was supported by a Wellcome Trust Prize Studentship and Mathematical Biology Training Fellowship. A.L. was supported by a Royal Society Fellowship. The Oxford part of the collaboration was funded by a Wellcome Trust Programme Grant, the Heidelberg part by the Max-Planck Gesellschaft. We are grateful to Sir David Cox for his comments on the statistics, to K. Stratford, M. Häusser, D. Flitney, M. O'Neill, S. Gough, G. Stuart, N. Spruston, P. Stern, and K. Bauer for their help and useful discussions, and to $M$. Kaiser for technical assistance.

Correspondence should be addressed to Guy Major, University Laboratory of Physiology, Parks Road, Oxford OX1 3PT, United Kingdom.

Copyright (C) 1994 Society for Neuroscience $0270-6474 / 94 / 144613-26 \$ 05.00 / 0$
}

Many dendrites are thought to possess a variety of active membrane conductances (e.g., Hounsgaard and Midtgaard, 1989). Under certain conditions, however, they may still behave, or can be made to behave, in a predominantly passive manner, for example, close to resting membrane potential in in vitro slices, or after "washout" of conductances or after application of channel blockers. Even when models incorporate active conductances, reasonably accurate estimates of the underlying passive "electrical skeleton" are necessary. For example, isopotential spheres can behave very differently from distributed branching cables with the same density of active conductances. Passive cable models can also be used as a reference against which to assess the effects of active conductances.

Cable properties have relevance for neural network models: most of these use "point" units with one electrical compartment (e.g., Rumelhart and McClelland, 1986). Studies in a variety of neurons suggest, however, that most are distributed cables and are capable of generating postsynaptic potentials (PSPs) of very different shapes (e.g., Rall, 1964, 1967; Rall et al., 1967; Jack et al., 1971; Turner, 1988; Stratford et al., 1989; Major, 1992; Nicoll et al., 1993). Units with such properties are particularly useful in networks learning temporal sequences (e.g., Tank and Hopfield, 1987; de Vries and Principe, 1992; Bressloff, 1993).

In this article we present a study of the passive cable properties of CA3 pyramidal neurons, including a detailed description of the modeling methods (also see Major, 1992). We pay particular attention to the effects of noise and pipette artifacts. Our objectives are (1) to describe a general method for establishing likely parameter ranges for passive cable models of neurons, using direct fitting of model to experimental responses and comparison of fit residuals with equivalent noise controls; (2) to apply this to CA3 pyramidal cells in in vitro slices (following Brown et al., 1981; Turner, 1984); and (3) to illustrate how these cells can generate differently shaped PSPS and postsynaptic currents (PSCs) at the cell body (e.g., Johnston and Brown, 1983; Rall and Segev, 1985; Williams and Johnston, 1991; Jonas et al., 1993) in response to identical plausible synaptic currents at different dendritic locations.

Many passive cable modeling studies to date have made use of the equivalent cylinder simplified representation (Rall, 1959). This may have been justifiable in the absence of good morphological data contradicting its main assumptions. These include (1) that a cell's dendrites should have equal electrotonic lengths, and (2) that its branching should obey the $3 / 2$ rule for diameters. More recently it has been shown that many neurons possess dendrites with different relative electrotonic lengths, assuming uniform dendritic $R_{m}$ (specific membrane resistance) and $R_{i}$ 
(cytoplasmic resistivity). Most pyramidal neurons, for example, have apical tuft dendrites that terminate, in electrotonic terms, farther from the soma than do the basal and apical oblique dendrites (e.g., Stratford et al., 1989; Larkman et al., 1992). Furthermore, at apical trunk branch points, the $3 / 2$ rule is violated (e.g., Hillman, 1979; Larkman et al., 1992). For these reasons it seems sensible to use models based on the best available morphological data. Because of variability between cells, the approach adopted here and in a number of other studies has been to combine electrophysiological and morphological measures from the same cell (e.g., Barrett and Crill, 1974; Durand et al., 1983; Fleshman et al., 1988; Clements and Redman, 1989; Stratford et al., 1989; Nitzan et al., 1990; Jonas et al., 1993; Rapp et al., 1993). Care is taken to include dendritic spines, which account for approximately half of a typical pyramidal cell's surface area (e.g., Larkman, 1991), and also the local axon arbors, because in CA3 pyramidal cells they are so extensive (see below).

Transient responses can yield more information about electrotonic architccturc than do steady-state responses, because of charge redistribution (e.g., Rall, 1969a). Furthermore, in nonisopotential cells, steady-state input resistance may be an unreliable measure because it is hard to determine how much of it is due to the recording electrode (Park et al., 1983; Wilson and Park, 1989), and in any case it is relatively insensitive to the value of $R_{i}$ (e.g., Nitzan et al., 1990). The test input used here is the short current pulse (Iansek and Redman, 1973). The early (charge redistribution) components of a short pulse response are relatively more prominent than those of its (approximate) time integral, the current step. Another advantage is that, for a given signal-to-noise ratio, short pulse responses tend to display better linear scaling with the magnitude and polarity of the injected current than do step or long pulse responses (e.g., Durand et al., 1983), because the membrane potential deviates markedly from its resting level for less time, and so has less chance to change slow voltage-dependent conductances.

It is not clear whether voltage clamp or voltage recording is the preferable mode for measuring cells' responses. Final time constants are faster under voltage clamp than under voltage recording with the same electrode, so it should be possible to average more responses over a given time. Noise due to spontaneous synaptic inputs may be reduced under voltage-clamp conditions, partly because of greater cable attenuation, and also because partial voltage control down the dendrites may reduce the boosting effects of some dendritic active conductances. Against these advantages is the additional noise introduced by the voltage-clamp amplifier itself, and the fact that it is possible to block active conductances and spontaneous synaptic events pharmacologically. Voltage clamp also introduces an extra model parameter (series resistance) that can never entirely be compensated for or avoided electronically. With tight-seal wholecell pipettes, voltage recording has an additional important advantage: the final time constant of a passive response should be the same as the membrane time constant, if the latter is uniform. We have therefore chosen the voltage-recording mode for this study, as being simpler and more direct than voltage clamp.

A number of techniques are available for simulating transient responses from arbitrarily branching cables: compartmental models (e.g., Rall, 1964; Hines, 1984, 1989; Segev et al., 1985; Clements, 1986; Holmes et al., 1992), segmental cable methods (e.g., Butz and Cowan, 1974; Turner, 1984; Holmes, 1986b), and the separation of variables analytical solutions (Major et al., 1993a,b; Major and Evans, 1994) used in this study.

Because the fitting of exponentials is inherently sensitive to cven small amounts of noise in the data (e.g., Lanczos, 1957; Holmes et al., 1992), we follow Clements and Redman (1989) and use direct fitting of model responses to their experimental counterparts.

Another problem that has not been addressed adequately in previous studies is how to establish likely confidence intervals for model parameters. A large part of our article is concerned with this issue. An optimal fit model may be substantially different from the correct model, for a variety of reasons, for example, (1) incorrect assumptions, (2) systematic errors, (3) random errors, and (4) nonuniqueness.

(1) Assumptions (for review, see Rall, 1977). The main ones made here are that the membrane is passive (with resistance and capacitance only); the cytoplasm is purely resistive (no reactance); the specific electrical parameters $C_{m}$ (specific membrane capacitance), $R_{i}$, and axodendritic $R_{m}$ are uniform throughout the cell; radial charge flow is negligible (Rall, 1969b); the extracellular medium is effectively isopotential; the currents flowing are too small to cause ionic depletion or accumulation (cf. Qian and Sejnowski, 1989); the recording is stable (i.e., no progressive washout of conductances or change in leak around the electrode occurs). We explicitly check for linearity and stability by superimposing scaled responses to different currents (see below); an objective test for this precaution is also developed (see Modeling methods). A subtle danger is that some active conductances, for example, those underlying sag, can result in apparently linear scaling; one countermeasure is to inspect semilog plots of responses for sag (e.g., Fleshman et al., 1988; see below).

(2) Systematic errors. We exclude calibration errors by using artificial neurons, and we record and simulate pipette artifacts (see below). In some cases, simulated pipettes are included in the models, because of nonsumming interactions between cells and pipettes. Morphological errors are considered in the Discussion.

(3) Random errors. The high-frequency component of recording noise is largely random and uncorrelated over time, and can be removed by filtering. In this study we filter the later, slower parts of responses more strongly than the earlier faster components (see below). Unfortunately, the lower-frequency component of the noise is due to recording instabilities and spontaneous synaptic inputs (the latter we minimize pharmacologically), and thus is highly correlated over time. In single sweeps, the correlation coefficient of noise at time $t$ to noise at time $t^{\prime}$ decays with time lag $t^{\prime}-t$ in a roughly exponential manner, with a time constant approximately equal to the slowest time constant $\tau_{0}$ of the cell or model (not shown). Its value at zero lag is typically around 0.6 for the cells in this study, after $5 \mathrm{kHz}$ filtering. Most conventional statistical methods assume random Gaussian noise that is independent at different time points (e.g., Landaw and DiStefano, 1984, p R668; Press et al., 1988, p 519), and so they cannot be applied here. When noise is correlated over time, it is still possible to reject fits and obtain parameter confidence intervals using $\chi^{2}$ statistics based on the multivariate normal distribution, providing the noise is approximately Gaussian (e.g., Diggle, 1990, Appendix B). These techniques, however, are complex (see Discussion). Because of remaining doubts about the validity of all the model assumptions, and because of the possibility of undetected systematic 
errors, we adopt a simpler and cruder (but possibly less sensitive) technique for fit rejection. A band is placed around the target data, within which the true noise-free response would fall about $95 \%$ of the time, and fits that escape from this band are rejected (see Modeling methods). The confidence level of the test is validated using both experimental and simulated noise.

(4) Nonuniqueness. There are (at least) two kinds of nonuniqueness: (a) models that yield the same predictions but have different parameters-"raw" electromorphological parameter trade-offs (e.g., Holmes and Rall, 1992) resulting in the same underlying "core" electrotonic representation of the model (Major et al., 1993a; Major and Evans, 1994); into this category fall certain kinds of morphological measurement error that we attempt to assess below; and (b) models that yield different predictions (core electrotonic model nonuniqueness) - into this category would fall models whose fits to the target data are indistinguishable by any objective criterion (e.g., Stratford et al., 1989; White et al., 1992), but that can result in very different predicted PSP shapes and amplitudes for given input locations. The most troublesome cause of this kind of nonuniqueness is the possible presence of a somatic shunt conductance $g_{\text {shunt }}$ of unknown magnitude, probably in part impalement-induced (e.g., lansek and Redman, 1973; Pongracz et al., 1991; Rall et al., 1992; Staley et al., 1992). In this study, we minimize the possible shunt by using whole-cell recording with seal resistances above $5 \mathrm{G} \Omega$. In addition, we attempt to estimate the seal resistance at the end of the recording by withdrawing the pipette slowly until an outside-out patch is formed. Because this does not fully eliminate the possibility of a somatic shunt (perhaps because of membrane dimpling or natural causes such as locally high conductance densities at the soma), we also explore the effects of nonzero shunts explicitly. Fortunately, the possible range of shunts is severely constrained by the high input resistances of the cells with whole-cell recording (see Results).

The majority of passive modeling studies make the assumption that $C_{m}-1 \mu \mathrm{Fcm}^{-2}$. Partly because of the possibility of morphological errors that can trade off with $C_{m}$ (e.g., Stratford et al., 1989; Holmes and Rall, 1992; Major et al., 1993a; Major and Evans, 1994), and partly because of direct evidence that the true value of $C_{m}$ is closer to $0.7 \mu \mathrm{Fcm}^{-2}$ (e.g., Fricke, 1931; Schwan and Carstensen, 1957; Fettiplace et al., 1971; Takashima and Schwan, 1974; Benz et al., 1975; Takashima, 1976; Haydon et al., 1980), we explore a range of $C_{m}$ values.

The traditional $R_{i}$ value for motoneurons of around $70 \Omega \mathrm{cm}$ (Barrett and Crill, 1974; Clements and Redman, 1989) is also abandoned here; much higher values of $R_{i}$ seem necessary for acceptable fits (e.g., Shelton, 1985; Stratford et al., 1989; Jonas et al., 1993; Rapp et al., 1993; Thurbon et al., 1994). Great care is taken here to eliminate the effects of pipette artifacts as a possible cause.

\section{Materials and Methods}

\section{Experimental methods}

Experiments were carried out in Heidelberg, reconstruction and modeling in Oxford. Four cells (CA3_15, CA3_16, CA3_32, and CA3_33) are considered.

Preparation and recording. For a detailed description of the method of slice preparation and whole-cell recording, see Hamill et al. (1981), Edwards et al. (1989), and Jonas et al. (1993). In brief, 19-21-d-old Wistar rats were decapitated, and the brains were removed rapidly and placed in ice-cold artificial cerebrospinal fluid (ACSF; see below). Transverse hippocampal slices $300 \mu \mathrm{m}$ thick were cut, placed on a net sub- merged in ACSF in a holding chamber, allowed to recover for half an hour, and then kept at room temperature $\left(20-24^{\circ} \mathrm{C}\right)$ for up to $5 \mathrm{hr}$.

Single slices were transferred into the recording chamber at room temperature and were continuously superfused $(\approx 2 \mathrm{ml} / \mathrm{min})$ with ACSF. A platinum frame supporting parallel nylon threads was used to immobilize the slice (Edwards et al., 1989). One pair of threads was farther apart than the others; the grid was placed so that one of these pinned down the fornix, and the other ran from CA4 to CA1, avoiding damage to $\mathrm{CA} 3$.

Borosilicate patch pipettes ( $2 \mathrm{~mm}$ outer diameter) were fire-polished directly before use. Thick $(0.5 \mathrm{~mm}$ ) walled pipettes (resistances of 4-5 $\mathrm{M} \Omega$ ) were used for the first two cells; thin $(0.3 \mathrm{~mm})$ walled pipettes (resistances of $3.5 \mathrm{M} \Omega$ ) were used for the last two, coated with Sylgard 184 (Dow Corning, Midland, MI), with fluid levels as low as possible both within and outside the pipette, to minimize transmural capacitance.

The ACSF composition was (mM) $\mathrm{NaCl}, 125 ; \mathrm{NaHCO}_{3}, 25 ; \mathrm{KCl}, 2.5$; $\mathrm{NaH}_{2} \mathrm{PO}_{4}, 1.25 ;$ glucose, $25 ; \mathrm{MgCl}_{2}, 1 ; \mathrm{CaCl}_{2}, 2 ;$ bubbled with carbogen $\left(95 \% \mathrm{O}_{2}, 5 \% \mathrm{CO}_{2}\right), \mathrm{pH} 7.2$ at room temperature. After a whole-cell recording was established, the following neurotransmitter blockers were added to the ACSF to block spontaneous synaptic potentials and to improve the signal-to-noise ratio $(\mu \mathrm{M})$ : $100 \mathrm{APV}$ (D-2-amino-5-phosphonopentanoic acid; Tocris Neuramin, Bristol, UK), 30 CNQX (6cyano-7-nitroquinoxaline-2,3-dione; Tocris), and 10 bicuculline (first two cells); or $100 \mathrm{APV}, 100 \mathrm{CNQX}, 100$ picrotoxin, 50 bicuculline, and 100 2-hydroxy saclofen (Tocris) (last two cells). The pipette solution contained (mM) K-gluconate, 120; biocytin, 13; KCl, 10; EGTA (ethyleneglycol-bis( $\beta$-aminoethylether) $N, N, N^{\prime}, N^{\prime}$-tetraacetic acid), 10; HEPES [ $N$-(2-hydroxyethyl)piperazine- $N^{\prime}$-(2-ethanesulfonic acid)], $10 ; \mathrm{MgCl}_{2}$, 2; $\mathrm{CaCl}_{2}, 2 ; \mathrm{Na}_{2} \mathrm{ATP}, 2 ; \mathrm{pH}$ adjusted to 7.3 with $\mathrm{KOH}$, total osmolarity of $290 \mathrm{mOsm}$, passed through a $0.2 \mu \mathrm{m}$ filter. All drugs and chemicals were obtained from Sigma (Deisenhofen, Germany), unless otherwise specified.

An EPC-7 amplifier (List, Darmstadt, Germany) was used to record from the first two cells, and an Axoclamp-2A (Axon Instruments, Foster City, CA) for the last two. Unlike the EPC-7, the Axoclamp-2A allowed pipette capacitance neutralization and resistance compensation in voltage recording mode, and could inject accurate square current pulses. Voltages were filtered at $5 \mathrm{kHz}(-3 \mathrm{~dB})$ and digitized at $10 \mathrm{kHz}$, and averages were stored to hard disk. Single sweeps from the last two cells were also recorded with an FM tape recorder.

Slices were viewed using a $40 \times, 2 \mathrm{~mm}$ working-distance water-immersion lens (Nikon, Tokyo, Japan) on an upright Zeiss (Augsburg, Germany) "standard 14" microscope with Nomarski optics. To obtain a cell with an intact dendritic tree, a soma was exposed at the bottom of a 100-150- $\mu \mathrm{m}$-deep crater in the CA3 cell body layer, using a cleaning pipette. A patch pipette was lowered onto the soma with minimal positive pressure, to reduce extracellular biocytin staining. After formation of a seal of over $5 \mathrm{G} \Omega$, whole-cell configuration was achieved using brief suction pulses. All cells had membrane potentials, without holding current, more negative than $-65 \mathrm{mV}$ (no tip potential correction).

Series resistance $R_{\text {ser }}$ increased with time; it was estimated in voltageclamp mode from $R_{\mathrm{ser}}=V_{i} / I_{p}$, where $I_{p}$ was the unfiltered current transient peak and $V_{t}$ was the size of the test pulse. In voltage-recording mode, with the EPC-7, $R_{\text {ser }}$ was also estimated from the height of the initial jump in voltage following a current step, and was below $15 \mathrm{M} \Omega$ at the end of the recording; the pipette capacitances were estimated to be $10-20 \mathrm{pF}$. With the Axoclamp- $2 \mathrm{~A}$, capacitance and resistance compensation were performed and periodically adjusted in the standard way, monitoring the unfiltered voltage response to a step current onset at high time resolution ( $R_{\text {ser }}$ ranges: $6-25 \mathrm{M} \Omega$ for CA3_32, and 7-9 M 2 for CA3_33; pipette capacitances $C_{\mathrm{pip}}$, about $2.5 \mathrm{pF}$ ).

The thresholds of all four cells were more than $20 \mathrm{mV}$ positive to rest, and the action potentials were typically $90-100 \mathrm{mV}$ in amplitude with half-widths of less than $2 \mathrm{msec}$. All the cells could be made to fire repetitively, but none demonstrated burst firing in response to somatic current pulses.

Each cell was held at about $-70 \mathrm{mV}$ (in all cases holding currents were less than $-0.1 \mathrm{nA}$ ). Once every $1100 \mathrm{msec}$ (first two cells) or 873.3 msec (last two cells), short pulses of current of amplitude -1 or +0.5 $\mathrm{nA}$ were injected after a baseline period of $200 \mathrm{msec}$, and the voltage response was recorded. The time integrals of the EPC-7 current pulses were measured after digitization at more than $80 \mathrm{kHz}$; ringing had no effect over the fit intervals chosen (see below).

Groups of 100 sweeps were averaged together on line, with selective 
rejection of occasional sweeps contaminated by large spontaneous voltage instabilities or unblocked synaptic events. After one to three consecutive averages, the current was changed to its other value, to allow linear scaling checks (see below) to be performed over the entire duration of the recording. Each cell was recorded from for about $30 \mathrm{~min}$, usually sufficient for 800-1200 sweeps. (Further action potentials were recorded from the first two cells after the short pulse responses, and showed no discernible change in threshold or shape.) The amplifier was switched back to voltage-clamp mode and the pipette was withdrawn gradually until a 5-10 G $\Omega$ outside-out patch was formed, except in the case of cell CA3_16, whose patch broke down. [Of the four cells, CA3_16 actually had the highest input resistance (see Results), so it is unlikely there was a substantial leak in this case.]

In the case of the first two modeled cells' pipettes, the patches were blown away and short pulse control responses were recorded in ACSF at approximately the original depths of the cells. From these responses, and from visual estimation of the submerged depth of pipette (about 2 $\mathrm{cm}$ ), the two pipette capacitances were estimated to be in the range of $10-20 \mathrm{pF}, 20 \mathrm{pF}$ giving the best agreement (with pipette simulations) to the controls, as well as being most consistent with the typical capacitance of $1 \mathrm{pF} / \mathrm{mm}$ for this kind of pipette (Purves, 1981, p 32). A number of additional pipettes of various resistances, both thick- and thin-walled (the latter Sylgarded), were pulled, filled to the same level and immersed to the same depth in ACSF as those of the last two cells. Extracellular controls were recorded both with and without capacitance neutralization. The pipette resistances tested spanned the range of measured series resistances.

The calibration of the recording apparatus and modeling software was checked using artificial neurons (resistor-capacitor networks), and computer models of them.

Histological processing. Following recording, slices were transferred immediately to $2 \%$ paraformaldehyde, $2.5 \%$ glutaraldehyde fixative and were left overnight at $4^{\circ} \mathrm{C}$. With some modifications, the biocytin reaction method of Horikawa and Armstrong (1988) was followed. The fixative and all other aqueous solutions were made in $0.1 \mathrm{M}$ phosphate buffer $(\mathrm{PB})$ at $\mathrm{pH} 7.4$. Each slice was resectioned to a thickness of 50 or $60 \mu \mathrm{m}$. After a $1-1.5 \mathrm{hr}$ detergent treatment in $2 \%$ Triton X-100, the subsections were incubated (room temperature) in HRP (horseradish peroxidase) conjugated to avidin (HRP-A; Vector Labs, Peterborough, UK) at a dilution of $1: 200$ in $1 \%$ Triton for $2-2.5 \mathrm{hr}$, with intermittent stirring. The subsections were rinsed three times in PB and transferred to a $0.16 \%$ solution of Hanker-Yates reagent for $15 \mathrm{~min}$ at $4^{\circ} \mathrm{C}$. Hydrogen peroxide solution was added to a final concentration of approximately $0.004 \%$, and the reaction was monitored under a stereomicroscope. When the injected cell had become sufficiently dark (about 5-15 min), the reaction was stopped by rinsing twice with $\mathrm{PB}$. The subsections were then osmicated in $0.25-0.5 \% \mathrm{OsO}_{4}$ solution for $10-15 \mathrm{~min}$, and then were rinsed in PB. After dehydration in graded ethanols, they were mounted in epoxy resin (E-mix, now "One-shot Embedding Kits," Fisons Scientific Equipment, Loughborough, UK) on microscope slides and coverslipped.

Morphology. Four cells with high-quality fills and electrophysiological data were selected. They were reconstructed from camera lucida drawings made with a light microscope (Nikon Optiphot) at $1000 \times$ magnification for the dendrites, using a $100 \times$ oil-immersion objective (numerical aperture, 1.25 ), and at $400 \times$ for the axons. See Larkman and Mason (1990) for a summary of terminology. Each segment was numbered, and the branching pattern of the cell was recorded.

The diameter $d_{j}$ (excluding dendritic spines or axonal boutons) of each segment $j$ was estimated at three points using an eyepiece graticule at a magnification of $1875 \times$, and the average was recorded to the nearest $0.1 \mu \mathrm{m}$ (nominally, because of the resolution limit of $0.2 \mu \mathrm{m}$ ). If a segment tapered substantially, it was split. All segments were assumed to be circular in cross section. The soma diameter was measured along its three major axes.

Lengths in the plane $\left(h_{j}\right)$ were measured on the camera lucida drawing, using a thin piece of insulated wire bent as accurately as possible along each segment $j$. A wiggle factor $\left(w_{i}\right)$ was estimated: (wire-measured length) $\div$ (straight line tip-to-tip length). Displacement $(z$,$) perpendicular$ to the plane of focus was measured with the calibrated fine-focus dial. The final length $l_{j}$ of a segment was given by

$$
l_{j}=w\left(h_{j}^{2}+z_{j}^{2}\right)^{1 / 2}
$$

where $w$ was the median wiggle factor for that kind of process, around $1.15 \times$ for dendrites; axons had more erratic courses, so had slightly higher $w$ factors. In this way $l_{j}$ was measured in all three dimensions, and shrinkage was, we hope, adequately accounted for; no additional correction was made for tissue shrinkage (see Discussion).

For those segments which lay reasonably close to a plane of focus (about half of the total), spines or boutons were counted. Graphs were made of spine (or bouton) density versus dendritic (or axonal) diameter. All segments for which no count was performed were assigned densities from these graphs. In general the very proximal segments had low spine densities for their diameters, so were not included in the plots, but were counted individually, as were the apical trunk segments.

The relatively thick apical trunk segments posed an additional problem: spines could be hidden behind the segment. The HRP-A reaction product appears slightly less dense than that resulting from HRP injection directly into cells, so it is just possible to estimate the number of spines occluded by a thick segment, since they appear as slightly denser dots. However, in the case of the thicker dendrites of the last two cells modeled, only spines sticking out either side were counted, and an occlusion correction factor was applied to give an estimate of the total density (adapted from Larkman, 1991, using the average spine dimensions in Harris and Stevens, 1989).

Single spine areas were assumed to be the same as for CAl pyramidal cells: $0.83 \mu \mathrm{m}^{2}$ (Harris and Stevens, 1989). The extra area associated with single boutons was estimated with the light microscope by measuring one medium-sized bouton on each of a large sample of axon segments of each cell.

The morphological data were converted into a form compatible with the programs described below. Spines and boutons were collapsed into their parent segments (Jack et al., 1989; Stratford et al., 1989; Larkman et al., 1992), multiplying each segment $j$ 's length by $F_{j}^{2 / 1}$ and diameter by $F_{j}^{\prime /,}$, where $F_{j}$ - (surface area with spines) $\div$ (area of shaft alone) for segment $j$. Segment $j$ 's morphotonic length $l_{M}=2 l_{j} / d_{j}^{1 / 2}$ is the morphological or "resistivity-free" component of its electrotonic length $L_{j}$ (i.e., it is the relative electrotonic length); $L_{j}$ itself can be calculated by dividing $l_{M}$ by $\Lambda=\left(R_{m} / R_{i}\right)^{1 / 2}$, the "morphoelectric factor" (Holmes and Rall, 1992; Larkman et al., 1992). A cutoff distance of $350 \mu \mathrm{m}$ before and $500 \mu \mathrm{m}$ after spine incorporation was used to separate apical obliques from the apical tuft. For all terminal segments of a given class, withincell unweighted means and standard deviations were calculated for diameters before spine incorporation, and diameters and soma-to-tip morphotonic distances after spine incorporation.

\section{Modeling methods}

Grand averages of short pulse responses. The modeling process will be illustrated using one cell, CA3_32. A similar procedure was applied to CA3_33. (Differences applicable to the first two cells will be noted when relevant.)

Data were redigitized off line from tape, in the same way as during the experiments, but capturing individual sweeps without averaging (not done for first two cells). All sweeps were inspected, and those contaminated with large noise artifacts were marked for rejection. All accepted responses were filtered with a $\sigma=0.05 t$ variable Gaussian digital filter, with $t=0$ at the pulse start. This procedure smooths out noise of frequencies much higher than those present in the response at a given time, filtering progressively more strongly at later times without distorting the underlying waveform (Fig. 1).

Figure 2 illustrates the procedure by which target fit transients were obtained:

(A) For each sweep the voltage baseline between 0 and $200 \mathrm{msec}$ (exclusive) was subtracted.

(B) Time $t=0$ was set to be the pulse start. 'I he response was scaled by $1 /$ (injected current) and passed through the $\sigma=0.05 t$ filter. All responses were thus normalized to an input current of $+1 \mathrm{nA}$.

(C) Groups of roughly 100 sweeps with the same original injected current were averaged into a "subaverage" and

(D) the standard error (SE) at each time point was computed

$(E)$ The subaverages were superimposed graphically to check for stability and linear scaling. They were also inspected for sag using logarithmic voltage axes (Fig. $2 E$, inset). An objective test of superposition was then performed on each possible pair of subaverages (see below).

$(F)$ Each subaverage from the group that superimposed adequately was assigned a weight equal to the absolute magnitude of its injected current, to maximize the final signal-to-noise ratio, and they were then combined into a "grand average." The variance var, of the voltage $V$, at the $i$ th digitized time point along each subaverage waveform was 
obtained from the standard error $\mathrm{SE}_{i}$, knowing the number of sweeps $n$ in the subaverage, using

$$
\operatorname{var}_{i}=n \times \mathrm{SE}_{i}^{2} .
$$

Rather than recomputing it from the single sweeps, the $\Sigma V_{i}^{2}$ term for the $i$ th time point was reconstructed using

$$
\sum V_{i}^{2}=(n-1) \operatorname{var}_{i}+n \bar{V}_{i}^{2}
$$

where $\bar{V}_{i}$ is the mean over all $n$ sweeps in the subaverage of the voltage at time point $i$ ( $i$ is not the index of a particular sweep). The $\Sigma V_{i}^{2}$ terms from all the relevant subaverages were weighted and summed together, as were the $\bar{V}_{i}$ terms and the $n$ values, and a new group weighted mean, variance, and standard error were calculated for each time point $i$. Despite increased filtering, SE tends to increase with time as the baseline recedes further into the past and slow noise events accumulate (Fig. $2 D, F)$. The grand average from each cell was used as the target waveform for fitting model responses (see below); the corresponding input current was $+1 \mathrm{nA}$. The number of sweeps making up the grand averages from the different cells is listed in Table 1.

In summary, the procedure for obtaining a grand average is as follows:

(Box 1)

REJECT VERY NOISY SWEEPS
$\downarrow$
EACH SWEEP, SUBTRACT PREPULSE BASELINE
$\downarrow$
SCALE BY 1 /CURRENT
$\downarrow$
VARIABLE GAUSSIAN FILTER EACH SWEEP
$\downarrow$
SUBAVERAGE WITH STANDARD ERRORS
$\downarrow$
SUPERPOSITION TEST
$\downarrow$
WEIGHT BY |CURRENT |
$\downarrow$
GRAND AVERAGE WITH STANDARD ERRORS

(In the case of CA3_15 and CA3_16, baseline subtraction, scaling and $\sigma=0.05 t$ filtering was performed on the subaverages, since individual sweeps had not been recorded. A grand average and approximate standard error time course for each of those two cells were estimated by treating those subaverages designated stable and passive as single sweeps; see below.)

Noise controls and confidence bands. A reasonably objective but sensitive test is required to allow some fits to be rejected and others to be accepted. If a fit residual (target - fit) deviates from zero $(V=0)$ by more than the deviation of (say) $95 \%$ of corresponding control baseline noise waveforms, then that deviation is unlikely $(<5 \%)$ to have occurred by chance alone: the fit is probably incompatible with the target data. A number of measures of deviation are possible: the one we have found to be most sensitive to large short-duration discrepancies between model and target (such as occur when $R_{i}$ or $C_{m}$ have the wrong values) is the maximum value of [residual $(t) \div \mathrm{SE}(t)$ ] over all time points $t$ in the fit interval. Another possibility is the root mean square of [residual $(t) \div$ $\mathrm{SE}(t)$ ] over the entire fit interval: the weighted fit SD (WSD). Unfortunately, this measure suffers from "dilution," that is, a short-lived serious misfit that is unlikely to have occurred merely by chance, combined with an extremely good fit over the rest of the waveform, can still lead to a WSD score that is not rejected. To some extent this problem can be circumvented by applying the WSD test to early parts of the response only, when trying to constrain $R_{i}$ and $C_{m}$. If the maximum [residual $\div \mathrm{SE}]<k$, this is equivalent to a zero $(V=0)$ line falling completely within a band of width $\pm k$ SEs around the noise (or residual) wavcform, or to the fit waveform falling within a $\pm k$ SE band around the target waveform.

For CA3_32 and CA3_33,400 msec control periods of baseline noise preceding the next current pulse were digitized from tape (Fig. $3 A$ ). The
Table 1. Fit details

\begin{tabular}{llcl} 
Cell & Interval $(\mathrm{msec})^{a}$ & Sweeps averaged & SEs $^{b}$ \\
\hline CA3_15 & $1-200$ & 700 & 3.7 \\
CA3_16 & $1-200$ & 1100 & 3.4 \\
CA3_32 & $3-200$ & 1000 & 3 \\
CA3_33 & $3-150$ & 400 & 3
\end{tabular}

"From starl of current pulse (duration, $0.5 \mathrm{msec}$ ).

"Giving, with corresponding noise control averages, approximately $95 \%$ confidence band for no escapes of voltage baseline extrapolated from preceding 200 msec period.

first $200 \mathrm{msec}$ was used as a baseline, and the subsequent $200 \mathrm{msec}$ was treated exactly as the responses themselves had been (Box 1), with variable Gaussian filtering from the new $t=0(200 \mathrm{msec}$ before next pulse), but omitting the superposition test. Note that the baseline was taken before the control noise interval, not during it (this would lead to smaller deviations). In the case of $\mathrm{CA}_{3}$ 32 32 , a slight response tail was corrected for.

The noise subaverages were plotted with bands of width $\pm k$ SEs about the mean, together with a horizontal line at $V=0$, which would correspond to the extrapolated prepulse baseline; $k$ was varied until the $V$ $=0$ line fell just within the band for the entire duration of an interval corresponding to the fit interval eventually used (3-200 msec for CA $3 \_32$; see below). The test is illustrated for CA3_32 in Figure $3 B$, using the noise subaverage that required the widest band to avoid escape of the zero line. If only $\pm 2.5 \mathrm{SEs}$ are used, the zero line escapes briefly at early times (arrow). With \pm 3 SEs, however, the zero line (or true baseline, if there were to be no noise) falls within the band for its entire duration. Figure $3 C$ shows an equivalent plot for the noise grand average; again the true zero line falls within the \pm 3 SE band around the actual mean.

Simulations were performed of averaged low-frequency noise. PSPs were produced at the outset by injecting double exponential currents of different kinetics at various locations along a single cylinder simplified representation of a basal/oblique arbor, using optimal electrical parameters close to those in the Results. Each noise sweep was generated by superimposing random numbers of model PSP waveforms (both excitatory and inhibitory) with different randomly selected time courses, amplitudes, and start times. A $200 \mathrm{msec}$ baseline period preceded the test period; sweep lengths were $800-1000 \mathrm{msec}$. Simulated high-frequency random Gaussian noise was not added since this had largely been filtered out of the experimental data. Over a wide range of conditions, if more than 100 sweeps were averaged, \pm 3 SEs gave a roughly $95 \%$ confidence band; that is, the zero line fell within the $\pm 3 \mathrm{SE}$ band about $95 \%$ of the time. The exact band width required for $95 \%$ confidence depended on the duration of the test interval, the membrane time constant, and the amount of noise. The cells in this study typically had time constants about $75 \%$ as long as the fit interval (see Results). Shorter test intervals required slightly narrower bands, although because of the slowness of the PSP waveforms, and hence the long-lasting correlations in the noise, the required band width was relatively insensitive to the fit interval-a distinct advantage of the band test over the WSD test above.

A more important result of the simulations was that the same width $k$ giving $\sim 95 \%$ confidence bands for subaverages (of $\geq 100$ sweeps) also appeared to give $\sim 95 \%$ confidence bands for grand averages (of 400 4000 sweeps). This has an intuitive explanation: because of the central tendency caused by averaging, one would expect the maximum residual (or noise value) to be proportional to $n^{-1 / 2}$ for a large number of sweeps $n$; since its $\mathrm{SE}$ also scales with $n^{-1 / 2}$, their ratio must stay roughly constant. For $n<30$ a small numbers effect became apparent: progressively wider bands became necessary to achieve a given confidence level.

In the case of real noise controls, all the subaverages (of about 100 sweeps) and the grand averages from CA3_32 and CA3_33 passed the $3 \mathrm{SE}$ test. From CA3_32, one of the 12 subaverages failed the $2.5 \mathrm{SE}$ tcst ( $92 \%$ passed). From CA3_33, all subaverages passed the $2.5 \mathrm{SE}$ test, and six of seven passed the 2 SE test (86\%). As with simulated noise, there appeared to be a small numbers effect with 30 or fewer averaged sweeps. 


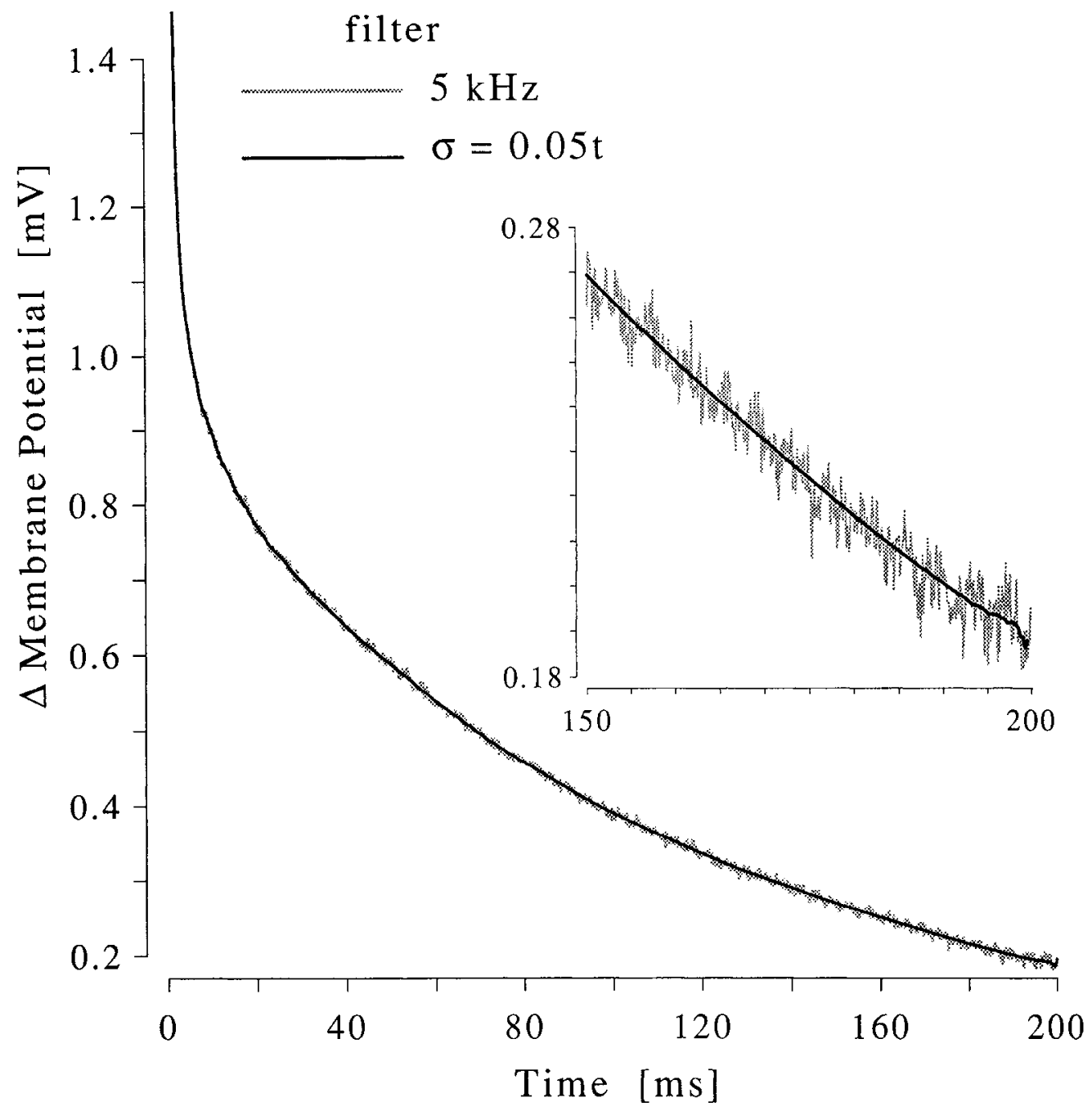

Figure 1. Variable Gaussian digital filtering. An average of 100 responses from CA3_32 is shown (gray, noisy line). Filtering with a $\sigma=0.05 t$ Gaussian results in the smooth black line. The signal-to-noise ratio is much improved (particularly at late times, when the filtering is heaviest; inset), without any appreciable distortion of the response.

Supcrposition tests. A further confidence band test, illustrated in Figure 4 , is now described for assessing whether two response waveforms superimpose adequately. The difference between two waveforms, $V_{1}-$ $V$, has a joint $\mathrm{SE}$ at each time point equal to $\left(\mathrm{SE}_{1}^{2}+\mathrm{SE}_{3}^{2}\right)^{\prime \prime}$. The differences were computed between all possible pairs of the simulated averaged noise waveforms described above, together with their joint SEs. The same width of band as for the original noise averages, \pm 3 SEs, was required to prevent the zero line escaping in about $95 \%$ of cases. This was also verified with the real noise controls: in the case of CA3_32, 65 of 66 possible subtracted pairs passed the 3 SE test $(98 \%)$. Three noise subaverages are shown in Figure $4 D$. The difference between subaverages 5 and 6 passes the 3 SE test (Fig. $4 E$ ), the typical case. The exception, the difference between subaverages 5 and 9 , just fails the test at early times (Fig. $4 F$ ). In the case of CA3_33, all 21 possible noise differences passed the 3 SE test.

Three of the subaverages from CA3_32 are plotted in Figure 4A. It can be seen that subaverages 4 and 5 superimpose extremely well; this is born out by the $3 \mathrm{SE}$ test on the difference waveform (Fig. $4 B$ ). Subaverages 1 and 4 appear very different by eye, and (Fig. $4 \mathrm{C}$ ) at late times (and very early times, before the fit interval start of $3 \mathrm{msec}$ ) the zero line escapes from the 3 joint SE band. Since this occurs in noise controls less than $5 \%$ of the time, it can be concluded that the difference is probably genuine, and not due to a chance deviation; the explanation is the sag at late times in subaverage 1 . The sag appears to have been washed out by waveform 3 (see Fig. $2 E$ ). Subaverages 3-12 superimpose well, and are used for the grand average (see above). In the case of

Figure 2. Procedure for computing grand average and $\sim 95 \%$ confidence band. $A$, Single sweep, showing voltage response to $-1 \mathrm{nA}$, duration 0.5 msec current pulse from cell CA3_32, filtered at $5 \mathrm{kHz}$ and digitized at $10 \mathrm{kHz}$. A large amount of high-and low-frequency noise can be seen. The average voltage over the $200 \mathrm{msec}$ baseline period preceding the pulse (injected at $200 \mathrm{msec}$; vertical arrow) is then subtracted, the response is scaled by $1 /$ current $=-1$, and $t=0$ is set to be the pulse start, to give the gray transient in $B$. $B$. High-frequency noise is removed without distorting the underlying response by digitally filtering with a variable Gaussian filter, standard deviation $\sigma=0.05 t$; result is black line. $C$, Seven consecutive $\sigma=0.05 t$ filtered sweeps are superimposed. Low-frequency noise, highly correlated over time, contaminates all responses and causes considerable intersweep variability. $D$, One hundred sweeps are averaged into a subaverage (solid line). The standard error at each time is multiplied by three to give a band (dashed lines) around the mean, in which the true response would lie about $95 \%$ of the time (see Fig. 3 for noise controls). E, All 12 subaverages from this cell are superimposed. Dashed and solid lines, $-1 \mathrm{nA}$ responses; dotted lines, $+0.5 \mathrm{nA}$ responses (all were scaled by $1 /$ current going from $A$ to $B$ ). All the subaverages, apart from the first two (dashed), superimpose reasonably well. The first two also show sag at late times (see inset semilog plot). An objective superposition test was performed (Fig. 4) to verify this impression. $F$, The last 10 subaverages are combined into a weighted grand average (solid), shown with its 3 SE band (dashed); the avcrage is less noisy than the subavcragc in $D$, and the 3 SE band is narrower, but the true response would still lie within the band about $95 \%$ of the time. 

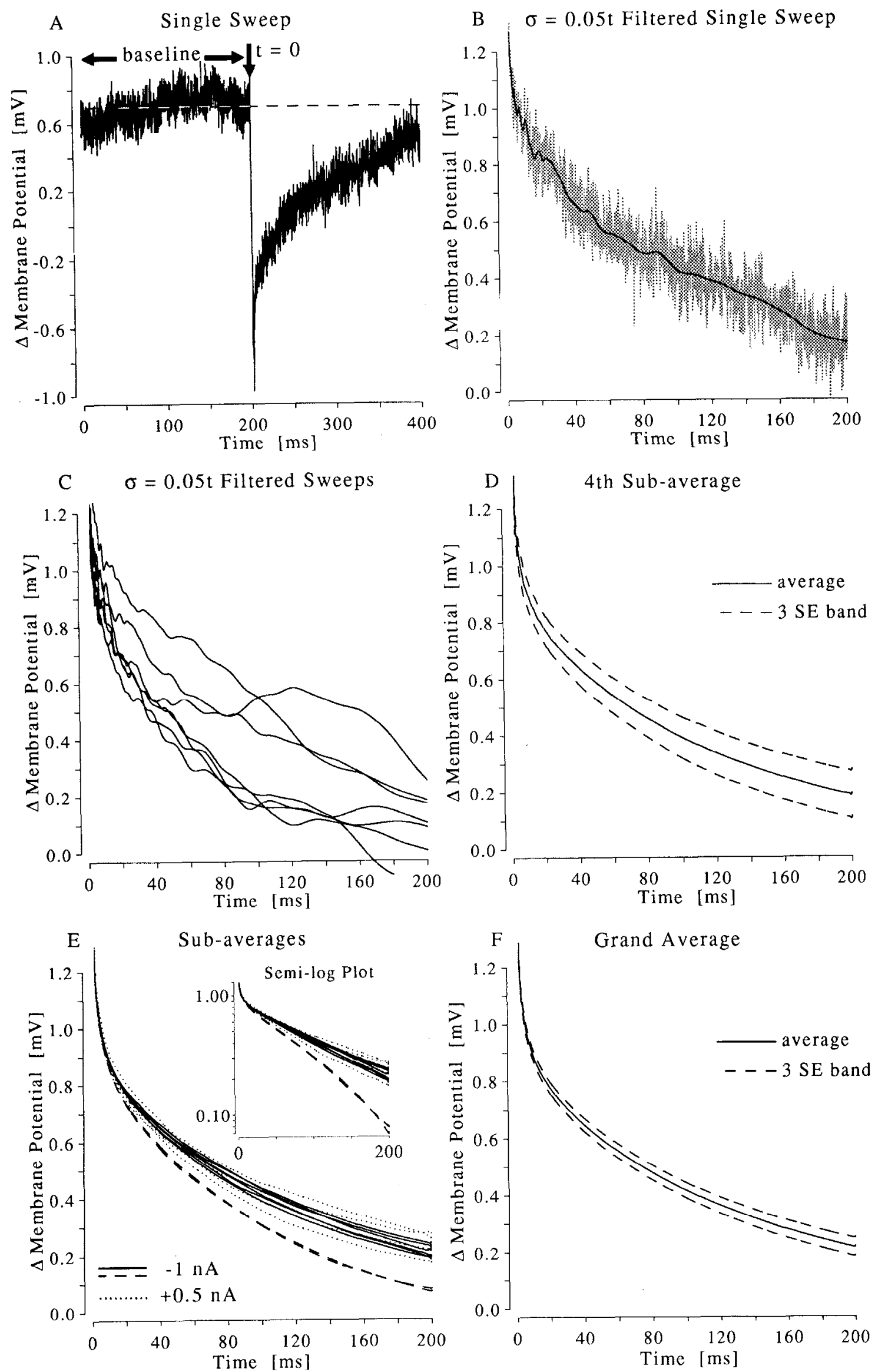
A Schematic Noise Protocol

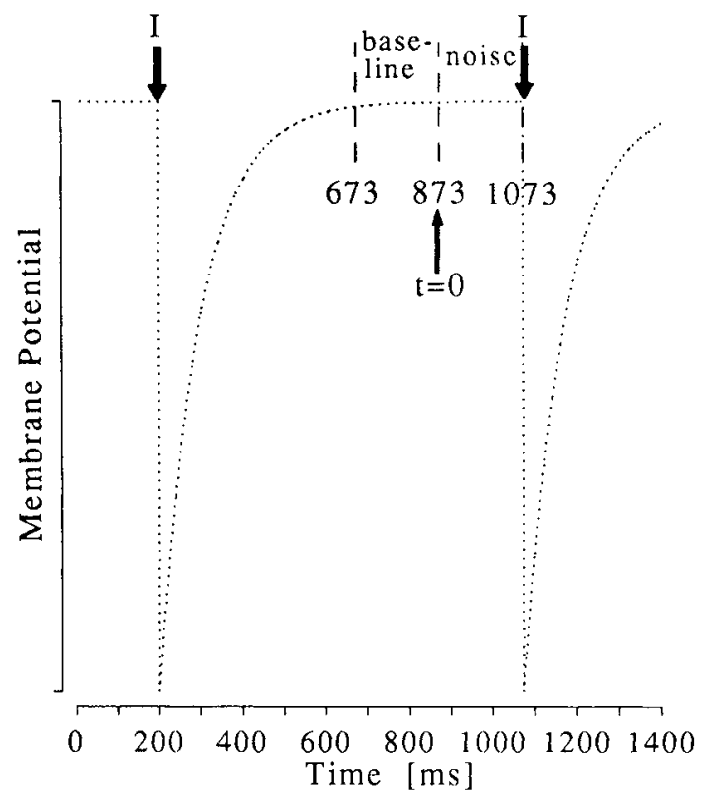

B
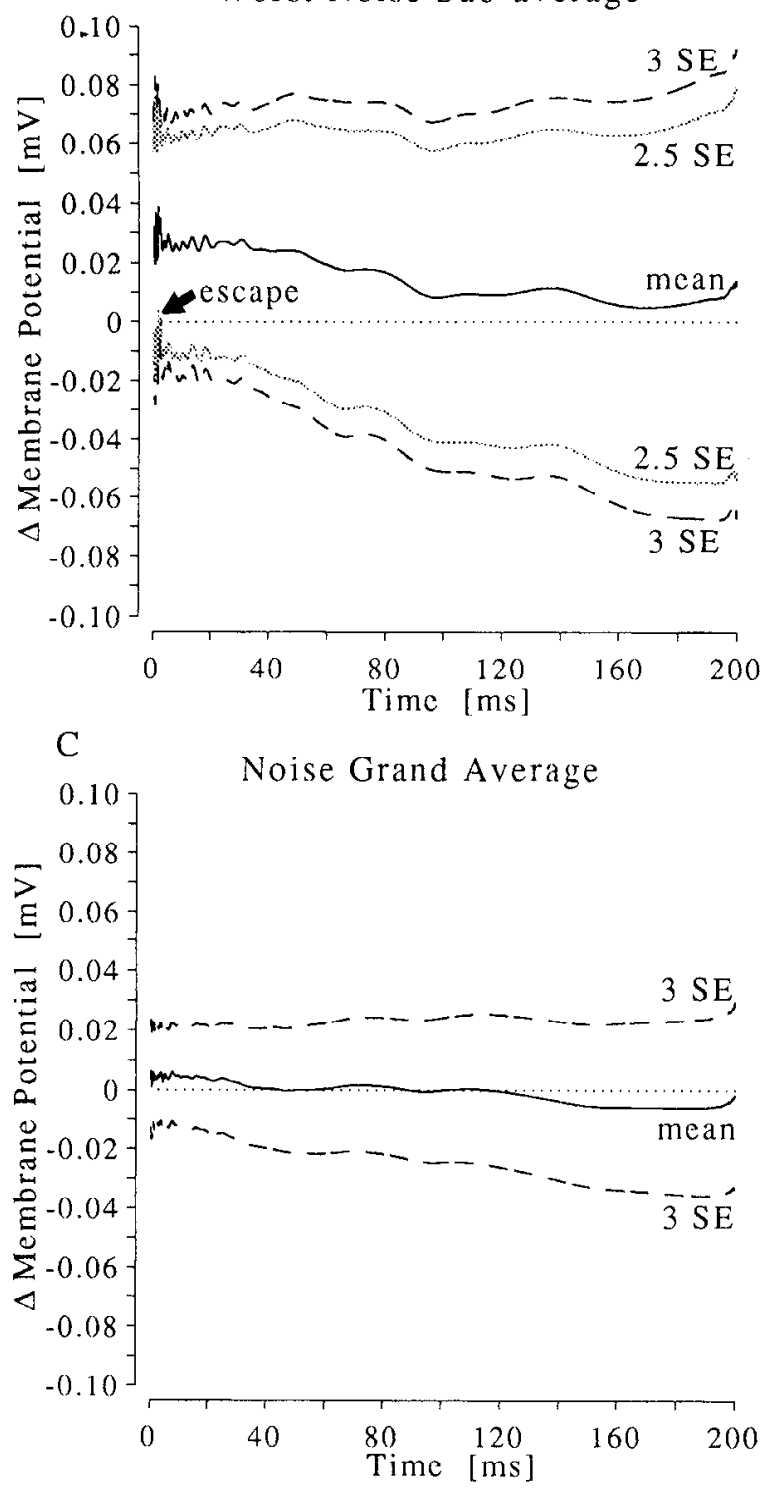

CA3_33, only subaverages 4-7 superimposed adequately. Subaverages 1-3 displayed varying degrees of sag.

Modifications of tests for CA3_15 and CA3_16. No objective superposition test was possible for the subaverages of CA3_15 and CA3_16, because no proper noise controls were available. Inspection of the superimposed waveforms with normal and semilog axes led to the exclusion of the first three subaverages of CA3_15, because of clear sag. No sag was evident on any of the subaverages from CA3_16; all were included in its grand average.

Because of the small numbers effect described above, grand averages were also computed for CA3_32 and CA3_33, treating the subaverages as single sweeps with zero $\mathrm{SE}$. The resulting approximate $\mathrm{SE}$ was usually smaller and more variable than the true SE (e.g., see Fig. $5 C$, below). The average ratio of approximate $\div$ true $\mathrm{SE}$ was approximately equal to the " $t$ ratio" given by the $\alpha=0.025$, degrees of freedom (df) $=n-$ 1 entry from a standard $t$ table (where $n$ is the number of subaverages) divided by the $\mathrm{df}=\infty$ entry. The following ad hoc procedure could convert the approximate SE into a closer (but imperfect) replica of the true SE: (1) filter SEs with a $\sigma=0.2 t$ variable Gaussian, to smooth out ripples; (2) multiply SEs by the appropriate $t$ ratio.

Equivalently, the width of the filtered approximate confidence band could be multiplied by the $t$ ratio, to improve its match to the true confidence band. Thus, instead of a 3 SE test, 3.7 SEs were required for CA3_15, and 3.4 SEs for CA3_16, to give a confidence level of $\sim 95 \%$.

Apparent slowest time constants, input resistances, and ends of fit intervals. The first subaverage and the grand average responses of each cell were analyzed. Each waveform was inspected using a logarithmic voltage axis, and a straight line was fitted by linear regression through the final decay phase, to obtain an estimate of the apparent time constant $\tau_{0}$ and the apparent amplitude $A_{0}$ of the slowest response component. (In the case of the grand average, the time at which the actual response deviated visibly below this line was chosen as the end of the fit interval; see Table 1.) The first apparent exponential component was peeled (Rall, 1969a) away from the response, and the process was repeated, until a time constant less than 1.5 times the maximum value of $\tau_{\text {nip }}$ for that cell was reached, where $\tau_{\mathrm{pip}}=C_{\mathrm{pip}} R_{\mathrm{scr}}$ is the pipette time constant, given a pipette capacitance $C_{\text {pip }}$ and a series resistance $R_{\text {ser }}$. (This cutoff is a crude way to avoid most of the pipette artifact.) The approximate input resistance $R_{N}$ was then calculated using Equation 5 of Durand et al. (1983):

$$
R_{\mathrm{y}}=I^{-1} \sum_{n=0}^{n_{\mathrm{nat1}}} C_{n}\left(1-e^{-w_{i} r_{n}}\right)^{-1}
$$

where $\tau_{n}$ and $C_{n}$ are the $n$th apparent time constant and amplitude, respectively, $n_{\max }$ ranged from 3 to 5 , and $I$ and $w$ are the amplitude and duration of the current. Measured apparent time constants and amplitudes were sensitive to the regression intervals chosen and were not taken to correspond precisely to the true exponential components of a particular cell's response (Holmes et al., 1992; Major et al., 1993a); overall $R_{s}$ was much less sensitive.

Fit interval start times and pipette artifacts. Unless otherwise stated, all times from now on are measured relative to the current pulse start. Extreme care was taken to avoid or account for pipette artifacts, because previous modeling has shown that pipette artifacts can boost early components of responses and raise the apparent $R_{i}$ required to fit the experimental data. Moreover, capacitative artifacts cannot simply be subtracted from the cell's response to eliminate their effects: the cell-pipette

Figure 3. Noise controls for fit rejection test. $A$, Schematic showing how noise control sweeps were obtained, by taking the $400 \mathrm{msec}$ directly preceding the next short pulse (arrow I), and using the first half as a baseline period. The final $200 \mathrm{msec}$ (from 873 to $1037 \mathrm{msec}$ ), or a subinterval of it, was taken as the test period: $t=0$ was set at $873 \mathrm{msec}$, and the procedure in Box 1 was applied. $B$, The most deviant noise subaverage (solid line) from CA3_32, plotted with 2.5 SE (standard errors; gray lines) and 3 SE (dashed lines) bands. The horizontal zero line (dotted) escapes from the $2.5 \mathrm{SE}$ but not from the $3 \mathrm{SE}$ band at early times (arrow). $C$, Noise grand average from CA3_32, plotted with 3 SE band (dashed). The zcro linc (dotted) stays within the band over the entire time shown, so the grand average passes the 3 SE test. Any fit residual failing the test would be extremely unlikely to have occurred by chance alone. 
interaction is nonsumming, the duration of the real artifact may outlast that of the extracellular control by a considerable margin (Major and Evans, 1994), since in whole-cell mode the pipette has to discharge to earth indirectly, via the cell and its distributed capacitances and resistances.

In Figure $5 A$, three extracellular pipette artifacts are shown, recorded with the Axoclamp-2A. The briefest, from a $3.3 \mathrm{M} \Omega$ pipette with optimal capacitance compensation ("cap. comp."), is effectively over within 0.1 msec of the pulse end. This response is better than could be expected from the $3.5 \mathrm{M} \Omega$ pipettes used to record from CA3_32 and CA3_33, whose series resistances during recording varied from 6 to 25 and 7 to $9 \mathrm{M} \Omega$, respectively. The $9 \mathrm{M} \Omega$ control lasts almost $0.5 \mathrm{msec}$ from the pulse end, which reduces to about $0.2 \mathrm{msec}$ with optimal cap. comp.

If the very early components of CA3_32's responses are inspected, however, it can be seen (Fig. $5 B$ ) that they increased throughout the recording (peaking during the ninth subavcrage), as the scrics resistance increased. This is reflected in the standard error time course (Fig. $5 C$ ): although the total SE is more or less steady by $1 \mathrm{msec}(0.5 \mathrm{msec}$ from pulse end), the inter-subaverage SE (computed by treating the subaverages as single sweeps) only reaches its minimum by $3 \mathrm{msec}$. This may in part be a consequence of the $\sigma=0.05 t$ filtering. To help make more objective decisions about safe fit start times, simulations were performed comparing cell-pipette combinations (Fig. 6) with the cells alone and the pipettes alone.

The pipette artifacts are assumed to be largely capacitative; judging from the extracellular controls, any residual polarization or other effects will probably be over by $0.5 \mathrm{msec}$ from the pulse end. Programs implementing analytical solutions for arbitrarily branching cables with nonuniform parameters were used (Major and Evans, 1994); all waveforms were identical to those from cquivalent compartmental models (adapted from Clements, 1986).

In the case of CA3_15, the no-shunt optimal fit model parameters $\left(C_{m}=0.65 \mu \mathrm{Fcm}^{-2}, R_{i}=210 \Omega \mathrm{cm}, R_{m}=180 \mathrm{k} \Omega \mathrm{cm}^{2}\right)$, found with the worst-case pipette ( $20 \mathrm{pF}, 15 \mathrm{M} \Omega)$, were used in simulations (see section Direct fitting, below). The pipette consisted of three segments with lengths and diameters as follows: 1 (tip), $500 \mu \mathrm{m}, 2 \mu \mathrm{m} ; 2,500 \mu \mathrm{m}, 4 \mu \mathrm{m} ; 3$ (wide end), $19 \mathrm{~mm}, 0.5 \mathrm{~mm}$. The local factors for multiplying the global electrical parameters were chosen to give a pipette capacitance of $1 \mathrm{pF} /$ $\mathrm{mm}, R_{\mathrm{scr}}=15 \mathrm{M} \Omega$, and a wall conductance of $<10^{-10} \mathrm{~S} / \mathrm{m}$ (i.e., $<2 \mathrm{pS}$ in all). Current was injected and recorded via the distal end of segment 3 , when a model included the pipette, and via the cell soma otherwise. The pipette tip was attached to the cell soma. For the extracellular control, the tip was voltage clamped to zero; that is, it was earthed.

The $1 \mathrm{nA}$, duration $0.5 \mathrm{mscc}$ short pulse responses are shown in Figure $6 \mathrm{~A}$. The pipette control is negligible by about $3 \mathrm{msec}$ from the pulse start, but the differences between the cell alone and cell + pipette responses are significant for the entire fit interval (1-200 msec), since the $A_{0}$ (slowest amplitude) terms of the two models $(0.916$ and $0.903 \mathrm{mV}$, respectively) differ by more than $1 \%$, and the slowest time constants $\tau_{0}$ (117 and $122 \mathrm{msec}$, respectively) differ by about $4 \%$. The $20 \mathrm{pF}$ pipette capacitance is an appreciable fraction $(\sim 4 \%)$ of the total cell capacitance $(\sim 530 \mathrm{pF})$. It is for this reason that the pipettes are included explicitly in the models of CA3 15 and CA3_16, with the entire range of likely pipette resistances and capacitances being explored (see below).

The pipette simulations for CA3_32 differed in that the length of segment 3 was varied between $1.5 \mathrm{~mm}$ and $10 \mu \mathrm{m}$, giving $C_{\text {pip }}$ between $2.5 \mathrm{pF}$ and $1 \mathrm{pF}$. The local $R$, factors in the pipette were adjusted to give $R_{\text {scr }}$ between 5 and $25 \mathrm{M} \Omega$. Only the $25 \mathrm{M} \Omega$ (worst-case) pipettes are illustrated here (Fig. $6 B$ ). The $C_{\text {pip }}$ measured experimentally was about $2.5 \mathrm{pF}$. Optimal capacitance compensation is simulated by reducing $C_{\text {pip }}$ to $1 \mathrm{pF}$ : it is hoped that this is (slightly) greater than the true effective residual capacitance; a more exact value would depend on knowing the rise time of the Axoclamp-2A measured with resistors of precisely known capacitance (Purves, 1981, p 49). It is not possible to reduce the residual capacitance to zero. The current pulse was $1 \mathrm{nA}$, duration $0.5 \mathrm{msec}$. The model had the no-shunt optimal fit parameters $C_{m}=0.79 \mu \mathrm{Fcm}^{-2}, R_{i}=270 \Omega \mathrm{cm}, R_{m}=200 \mathrm{k} \Omega \mathrm{cm}^{2}$, and the axon was omitted to allow the program to find smaller time constants. The uncompensated effective artifact lasts till about $2.5 \mathrm{msec}$, and the compensated artifact lasts till about $2 \mathrm{msec}$; the corresponding controls are essentially over by 0.9 and $0.6 \mathrm{msec}$, respectively, from the pulse start. The cell-alone and cell + pipette responses converge at late times because the pipette capacitance is a negligible fraction $(<0.5 \%)$ of the cell capacitance ( $\sim 530 \mathrm{pF}$, without axon).

The fit intervals chosen for both CA3_32 and CA3_33 commenced at $3 \mathrm{msec}$ from the pulse start, to avoid the most pessimistic effects of the pipettes. As can be seen below (Results), this was still enough to constrain $R_{i}$ reasonably well for both cells, although an earlier start time would have yielded a narrower range. The fit intervals for the first two cells (with models including pipettes) were started at $1 \mathrm{msec}$ (i.e., 0.5 msec after the end of the pulse), by which time the actual extracellular controls were over (not shown), in case these were due in part to noncapacitative artifacts.

Direct fitting. Following Clements and Redman (1989), a direct fitting program was used that called an implementation of the branching cable analytical solution (Major et al., 1993a) as a subroutine of a simplex optimization algorithm (Nelder and Mead, 1965; Press et al., 1988, pp 305-309). A + I nA, duration 0.5 msec short current pulse was injected into the soma of each model and the somatic voltage was recorded. The parameters to be optimized were $C_{m}, R_{i}, R_{m}$, and $g_{\text {shun. }}$. The cost function to be minimized was (WSD ${ }^{2}+0.5 n_{\text {escapes }}$ ), where WSD (defined above) is the weighted root mean squared deviation between the model and the target (grand average) waveforms over the fit interval, and $n_{\text {escapes }}$ is the number of points that escape from the $\sim 95 \%$ confidence band (intervals and SE levels are given in Table 1). Once a model response was within the target confidence band, that is, $n_{\text {escapes }}=0$, this cost function simplifies to its first term, and weighted least-squares fitting was performed. Outside the confidence band, the second term tended to dominate the cost function, causing the number of escapes to be minimized.

For CA3_15 and CA3_16, optimization was performed with current injection and voltage recording via a model pipette attached to the cell soma. A version of the fitting program was used that incorporated the analytical solution for nonuniform electrical parameters, fixing the resistances and capacitances in the pipette segments but varying the global specific electrical parameters of the cell (Major and Evans, 1994). Three pipette models were used for both cells: the most likely (10 M, $20 \mathrm{pF}$ ), best case ( $5 \mathrm{M} \Omega, 10 \mathrm{pF})$, and worst case (15 M, $20 \mathrm{pF}$ ).

For most fits, $g_{\text {shunt }}$ was fixed to zero; it was assumed that the seal resistance did not change significantly between cell-attached, whole-cell, and outside-out patch configurations (see Preparation and recording, above). One optimal fit was performed, allowing all three remaining electrical parameters to vary. Then each parameter in turn was fixed at progressively more extreme values (both above and below its optimum), until the constrained best fit was rejected by the confidence band test. This is illustrated for CA3_32 in Figure 7. In Figure 7A, a fit that is only just accepted is shown, grazing the inside of the $3 \mathrm{SE}$ target band of the grand average experimental response. It is just possible that this waveform plus experimental noise could have produced the actual recorded grand average. A fit that is rejected is shown in Figure $7 B: R$ is too high, and the early components rise above the upper edge of the 3 SE band; it is extremely unlikely that this waveform plus experimental noise could have led to the actual grand average. The procedure was repeated for all three pipette models in the case of the first two cells.

Because of the possibility that $g_{\text {shun }}$ might not be zero, either because of intrinsic somatic conductances, or because of an undetected leak that vanished when the outside-out patches were pulled, a final series of fits was performed (using only the most likely pipettes for the first two cells), allowing $g_{\text {shun }}$ to take a number of fixed values while the other parameters were optimized freely.

\section{Results}

The results of preliminary fits and voltage-clamp simulations using one of these cells (CA3_15) have already been published (Jonas et al., 1993). The optimal parmeters found in that study, which did not include the pipette in the model, were only slightly different from those listed below (without pipette: $C_{m}=+3.5 \%$, $R_{i}=+15 \%, R_{m}=-10 \%$ ).

\section{Morphological results}

All four cells were from the lower part of area CA3 (near CA4). All had extensive dendritic arborizations, with four main zones: (1) the basal arbor in stratum oriens, (2) a short apical trunk in stratum lucidum that branched into two or more secondary trunks, (3) apical oblique dendrites in stratum radiatum, and (4) an apical tuft in stratum lacunosum moleculare. A camera 

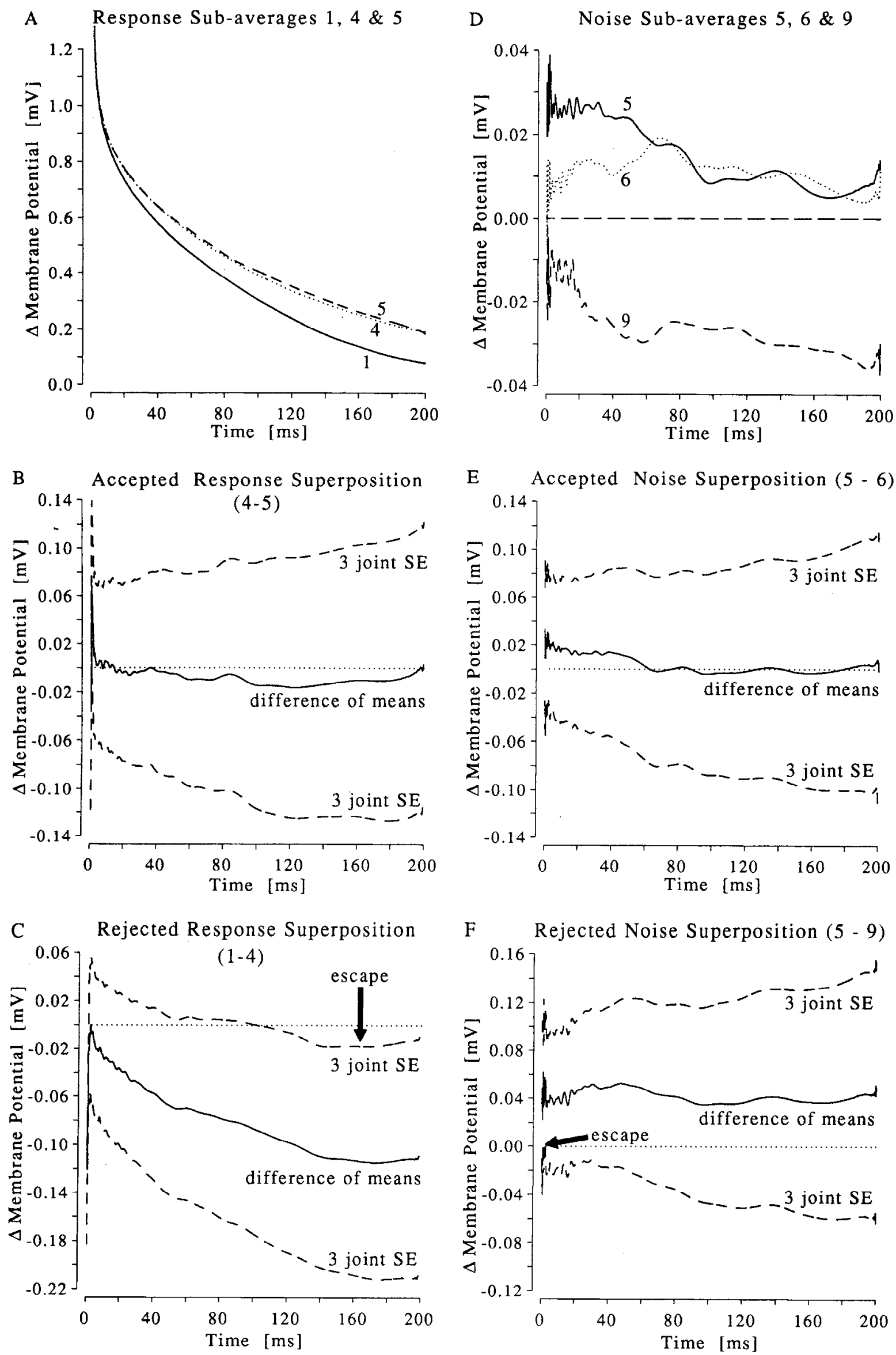
lucida drawing of the dendritic tree of CA3_33 is shown in Figure 8 , and a smaller scale drawing of the axon and dendrites of CA3_32 is shown in Figure 9. A photograph and camera lucida drawing of the dendritic tree of CA3_15 can be found in Jonas et al. (1993). Thorny excrescences were visible on proximal trunk segments, and other segments, apart from the most proximal basal segments, were densely covered by dendritic spines. The axon arbors of all four cells ramified for about 500 $800 \mu \mathrm{m}$ in either direction along stratum oriens and stratum radiatum, with numerous boutons. A few branches with boutons crossed stratum pyramidale and stratum lucidum. There were numerous amputated axonal branches at the cut surfaces of the slice, but few, if any, truncated dendrites.

Morphological measures from the four cells are summarized in Table 2. The average extra surface area associated with single boutons was estimated to be about $1 \mu \mathrm{m}^{2}$, which is compatible with the volumes quoted in Table 2 of Harris and Stevens (1989). The values varied slightly with the axon segment diameter. The mean spine density was about $3 \mu \mathrm{m}^{-1}$, which is higher than in adult visual cortical pyramidal cells (Larkman, 1991), but comparable to that in adult hippocampal CAl pyramidal cells (Andersen, 1990; Harris et al., 1992: electron microscopic measures). The bouton density increased approximately linearly with axon segment diameter in all cells (not shown), with an average value of about $0.3 \mu \mathrm{m}^{-1}$, or one bouton every $3.7 \mu \mathrm{m}$ along the axon. The cells had large numbers of dendritic spines: the mean number was about 30,000 . The mean number of boutons was over 5000 , and would have been much higher in vivo, before slicing amputated most of the axonal tree. Table 2 also gives terminal segment mean diameters before and after spine or bouton incorporation (the mean axon diameter is not changed to within one decimal place).

\section{Morphotonic or relative electrotonic structure}

Morphotonic dendrograms of all four cells are shown in Figure 10. The electrotonic scaling obtained from the optimal fit model of each cell (see below) is indicated at the top of the ordinate. The soma-to-tip morphotonic distances and summed (diameter $)^{3 / 2}$ values $\left(\Sigma d^{3 / 2}\right)$ for the terminal segments of each class of dendrite are given in Table 2. The morphotonic lengths of the apical trunks plus tufts are roughly twice those of the basal or oblique dendrites. In addition, the combined $\Sigma d^{3 / 2}$ of the basals and obliques ranges from about three to five timcs the $\Sigma d^{3 / 2}$ of the apical tufts. Therefore, the cells cannot be represented adequately as single cylinders.

\section{Linear scaling, stability, and washout of sag}

In the case of three of the cells, the first two or three response subaverages showed evidence of sag when inspected using logarithmic voltage axes (e.g., Fig. $2 E$, inset). This appeared to wash out reasonably quickly; later subaverages had no sag and superimposed well (as judged by eye and by confidence band tests). There was therefore no need to use channel blockers to "linearize" the cells (cf. Major et al., 1990; Rapp et al., 1993): once the sag was gone they appeared to behave passively.

\section{Input resistances and apparent time constants}

Apparent slowest time constants $\tau_{0}$ and approximate input resistances $R_{x}$ for the four cells, both "initially" and after washout of sag, are given in Table 3 . The former are estimated from the first subaverage, and the latter from the grand average of the linearly scaling, stable short pulse responses. The values are much higher than those for adult guinea pig CA3 cells recorded with sharp electrodes (mean $\tau_{0}=19 \mathrm{msec}, R_{N}=39 \mathrm{M} \Omega$ : Brown et al., 1981) and slightly higher than those obtained by the nystatin patch method (mean $\tau_{0}=66 \mathrm{msec}, R_{N}=135 \mathrm{M} \Omega$ at $-64 \mathrm{mV}$ : Spruston and Johnston, 1992). The differences could reflect age, species, membrane potential, or method. Certainly there is now thought to be a substantial somatic shunt with sharp electrode recording (see introductory remarks). The nystatin patch method may avoid the washout of sag and perhaps other conductances observed with these cells; indeed, the initial responses yield $\tau_{0}$ and $R_{A}$ values closer to those found by Spruston and Johnston (1992). Since in our experiments a few minutes in whole-cell mode always elapsed before the first short pulse responses were recorded, and since the sag disappeared within another $5 \mathrm{~min}$, it is likely that significant washout had already occurred before the "initial" subaverage. Apparent $\tau_{0}$ values before any washout may therefore have been similar to those found by Spruston and Johnston (1992). We should add that measurement of $\tau_{0}$ in the presence of strong sag may not be meaningful because there is no portion of the data that shows single exponential decay. The $\tau_{0}$ values obtained are very sensitive to the regression or fit interval chosen. The problem is to some extent obscured if traces are very noisy.

\section{Optimal fits and parameter ranges}

The optimal fits of all four cells (in the case of the first two, with the most likely pipette) are shown in Figure 11. The target data (dashed lines) and the model responses (dotted lines) superimpose so well that they are hard to resolve. The optimal fit parameters are summarized in Table 4 , along with the parameter extremes from all the constrained fits passing the confidence band test when systematic explorations were undertaken (3040 fits per cell).

The mean optimal $C_{m}$ of $0.75 \mu \mathrm{Fcm}^{-2}$ is surprisingly close to values measured directly (see introductory remarks). The mean optimal $R_{i}$ of around $270 \Omega \mathrm{cm}$ is several times higher than the traditional $70 \Omega \mathrm{cm}$. The lowest $R_{i}$ compatible with any of the cells was $100 \Omega \mathrm{cm} . R_{m}$ is around $120-200 \mathrm{k} \Omega \mathrm{cm}^{2}$ but this will

Figure 4. Superposition tests for response subaverages. Procedure for responses is illustrated on left; equivalent noise controls, on right. A, Three subaverages of $\sim 100$ sweeps from CA3_32. Subaverage 1 (solid line) does not superimpose well with subaverages 4 and 5 (dotted and dashed lines, respectively). $B$, The difference between subaverages 4 and 5 (solid line) is plotted together with a 3 joint SE band (dashed), obtained by summing the standard variances at each time point; the zero line (dotted) does not escape from the $3 \mathrm{SE}$ band over the entire interval shown: the superposition test is passed. $C$. Equivalent plot for the difference between subaverages 1 and 4 ; the zero line escapes at times (arrow) after about 100 msec, because of the sag in subaverage 1 (seen in Fig. $2 E$, inset): the superposition test is failed. The zero line also escapes at early times $\leq 0.8$ msec, perhaps because of changes in pipette series resistance. $D$. Control noise subaverages 5,6 , and 9 (solid, dotted, and dashed lines, respectively). Subaverages 5 and 6 , but not 9 , superimpose reasonably well. $E$. The difference between subaverages 5 and 6 (solid line) with their 3 joint SE band (dashed): the zero line (dotted) does not escape from the $3 \mathrm{SE}$ band for the entire interval shown. This is the case with $98 \%$ of the possible noise control difference waveforms. $F$. The one exception is the difference (solid line) between noise subaverages 5 and 9 ; the zero line just escapes at early times (arrow) from the 3 joint SE band (dashed). 

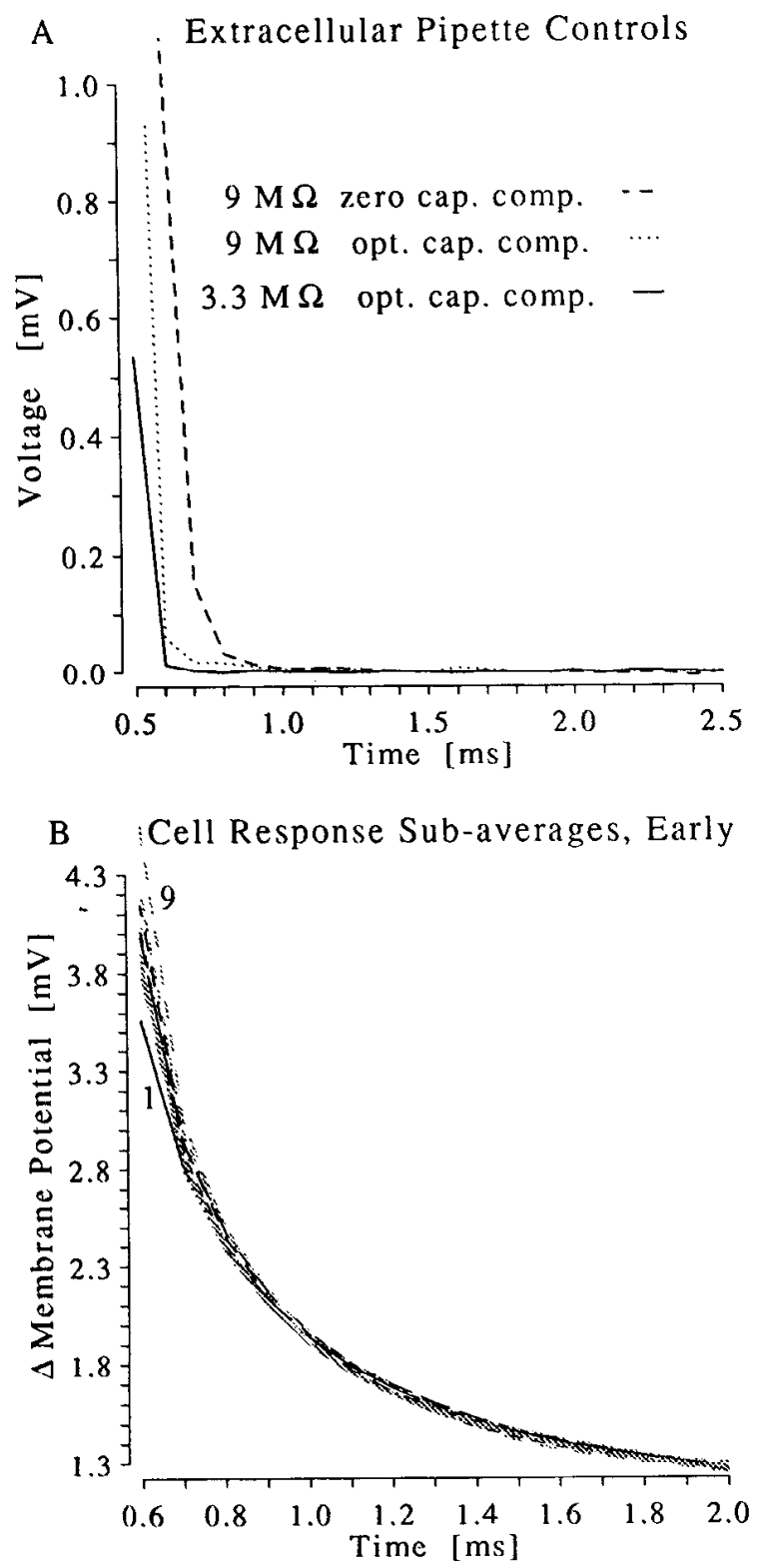

C Average of All Cell Sub-averages, Early

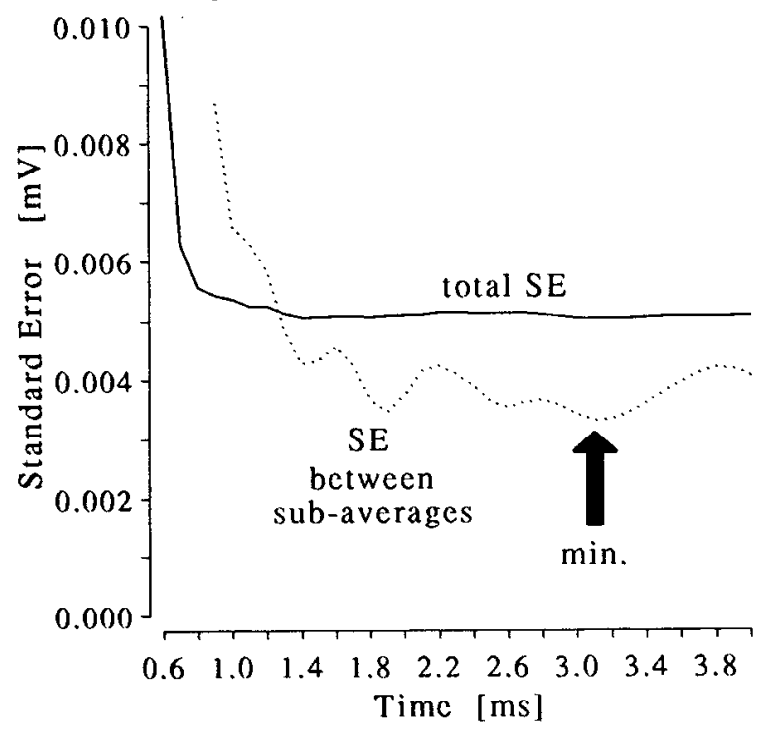

probably vary more from situation to situation (see Discussion). It is also the least reliable figure, if shunts are allowed (see below).

The fit WSDs (defined above) give an indication of overall goodness of fit; roughly $95 \%$ of WSDs of control noise subaverages, with respect to a $V=0$ line over the same fit intervals, were below 1.6. The noise simulations described above showed that for $n>100$ the distribution of noise WSDs was roughly the same for subaverages and grand averages (again, because both deviations and SEs are proportional to $n^{-1 / 2}$, where $n$ is the number of sweeps). Any fit with a WSD greater than 1.6 could have been rejected at the $95 \%$ confidence level. In practice, the confidence band test proved more sensitive, particularly with respect to constraining $R_{t}$. If the WSD test was restricted to the first $50 \mathrm{msec}$, then it constrained $R_{i}$ and $C_{m}$ about as well as the confidence band test.

The morphoelectric factor $\Lambda$ varied between 17 and $34 \mathrm{~cm}^{1 / 2}$ for the optimal models. Its overall range was $17-42 \mathrm{~cm}^{1 / 2}$, leading in all cases to extremely compact steady-state electrical geometry (see below). There may be increases in membrane conductances with development, which would cause $\Lambda$ in adult neurons to be lower.

\section{Fits with nonzero shunts}

When the shunt was allowed to vary away from zero, nonuniqueness became apparent: the optimal fits, shown in Table 5 , were hard to distinguish on the basis of their WSD scores, and a wide range of shunt models could not be rejected by the confidence band test (all those shown were accepted). In all cases but one, the optimal WSD score occurred at shunts of greater than zero. In short, there is no objective way of proving statistically that there was no somatic shunt in these cells; we need to rely on other arguments (in particular, the formation of outside-out patches upon withdrawal of the pipette). The maximum value of the shunt (to the nearest $\mathrm{nS}$ ) that allowed acceptable fits is shown in Table 5 for each cell. Unlike with sharp electrode recordings, the possible values of the shunt are severely constrained by the low input conductances of the cells (the worst case was CA3_33, which had the highest input conductance). The shunt can vary, essentially, from zero to the cell's total input conductance, and still yield models resulting in acceptable fits. The uncertainty over the values of $C_{m}$ and $R_{i}$ is not too large: shunts reduce the optimal values of both parameters slightly. However, the optimal valuc of $R_{m}$ is extremely ill con-

\footnotetext{
Figure 5. Real pipette artifacts. $A$, Extracellular controls for two thinwalled, Sylgarded pipettes submerged to the same depth as those used to record from CA3_32 and CA3_33. Solid line, 3.3 M 2 pipette, with optimal capacitance compensation (opt. cap. comp.); dotted line. $9 \mathrm{M} \Omega$ pipette, with opt. cap. comp.; dashed line, without cap. comp. (dial on zero position, neither enhancing nor neutralizing). The pipette capacitances were estimated to be less than $2.7 \mathrm{pF}$. B. First 2 msec of CA3_ 32 subaverages superimposed to show boosting of early components as series resistance creeps up from about $6 \mathrm{M} \Omega$ (subaverage 1, solid black line) to $25 \mathrm{M} \Omega$ (subaverage 9, gray, dashed). The other responses are either black or gray (last four), with different line styles. $C$, Standard error time course for CA3_32 "total" average at early times, prepared from all sweeps (total SE, solid line), and from all subaverages, treated as single sweeps (dotted). The latter variations only reach a minimum (arrowed) at about $3 \mathrm{msec}(2.5 \mathrm{msec}$ from pulse end). Notice also that the between-subaverage SE is lower, and more irregular, than the total SE. This obervation has implications for the width of the confidence bands for CA3_15 and CA3_16 (see Modification of tests for CA3_15 and CA3_16, in Modeling methods).
} 


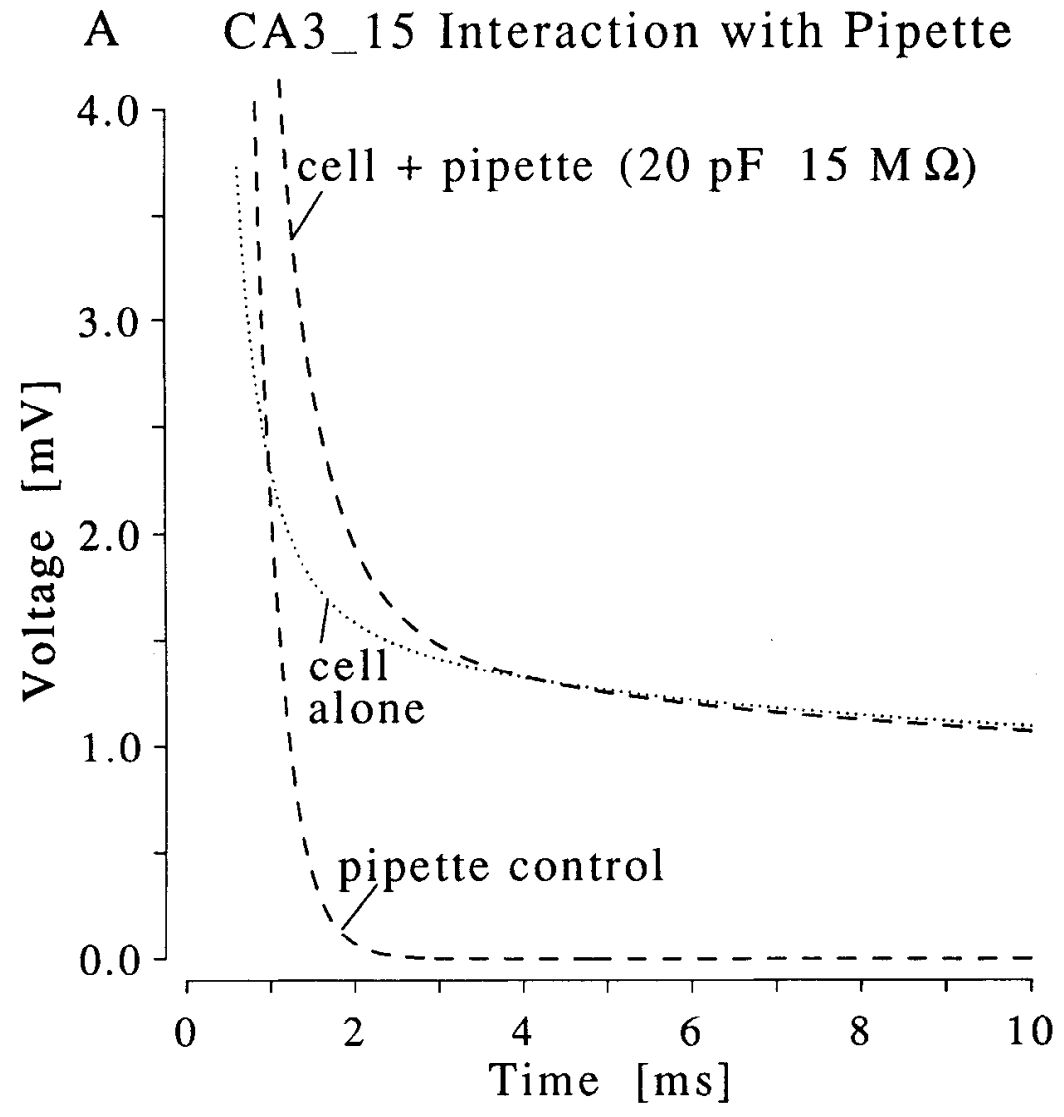

B CA3_32 Interaction with Pipette

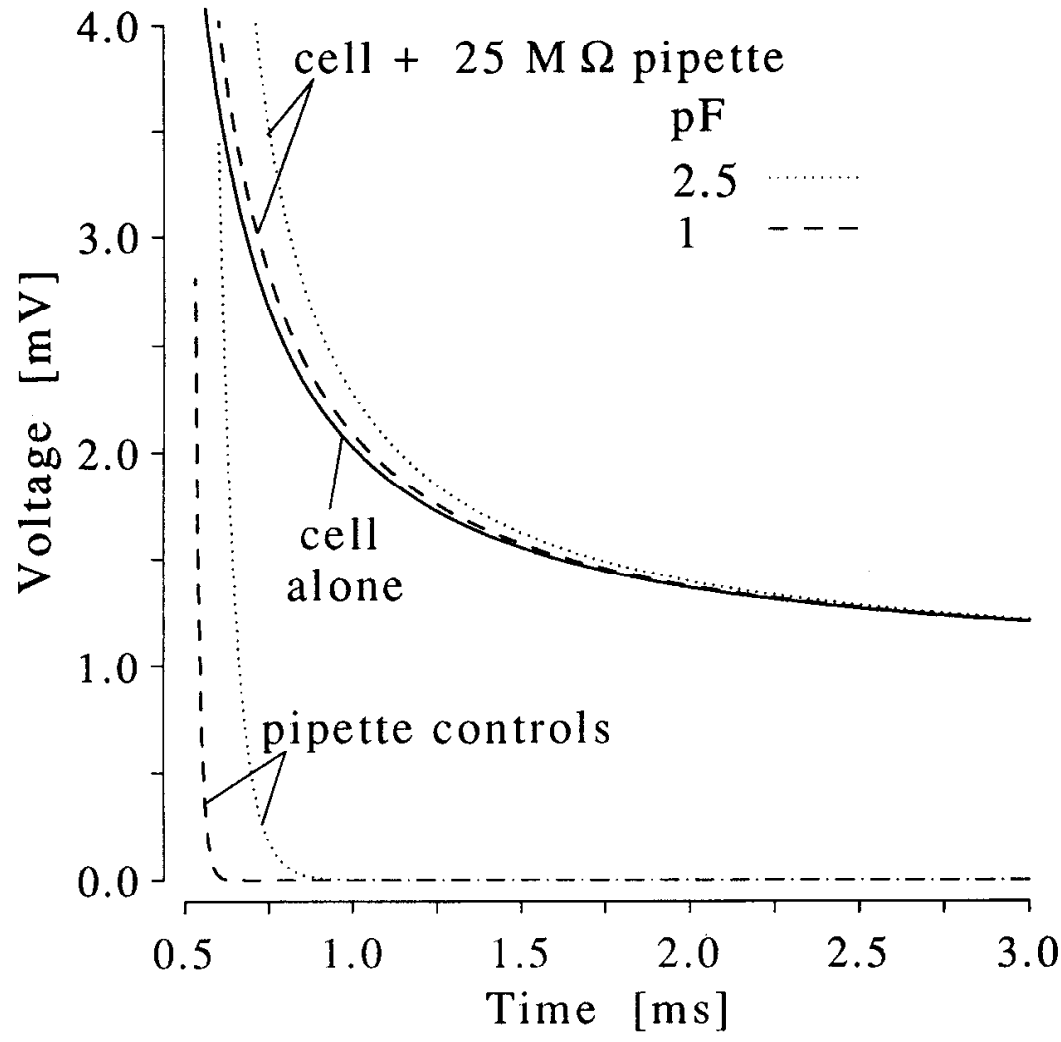

Figure 6. Nonsumming cell-pipette interactions. See Fit interval start times and pipette artifacts, in Modeling methods, for details. Simulations were performed using nonuniform analytical solution programs. $A, \mathrm{CA} 3 \_15$, optimal fit model short pulse response obtained recording via worst-case pipette (upper dashed line), compared with response of cell alone with same electrical parameters (dotted line) and extracellular pipette control response (lower dashed line). The deviation between the first two waveforms at early times lasts longer than the extracellular control, and there is a noticeable difference at late times for the entire fit interval (10-200 msec; not shown), because of the large pipette capacitance. $B, C A 3 \_32$, optimal fit model short pulse response (solid line) compared with that obtained recording via a $25 \mathrm{M} \Omega$ pipette (worstcasc resistance) with capacitance $C_{p i p}$ either $2.5 \mathrm{pF}$ (uncompensated) or $1 \mathrm{pF}$ (largely compensated). The corresponding extracellular pipette controls are also shown. Dotted lines, $C_{p i p} 2.5 \mathrm{pF}$; dashed lines, $C_{\text {pip }} 1 \mathrm{pF}$. The effective difference between the cell alone and the cell + pipette responses lasts much longer than the pipette controls, in the worst case requiring $3 \mathrm{msec}$ to become negligible $(2.5 \mathrm{msec}$ from pulse end). Because of the relatively low pipette capacitance compared with the cell's capacitance, the first three waveforms converge at late times. 
A Accepted Fit
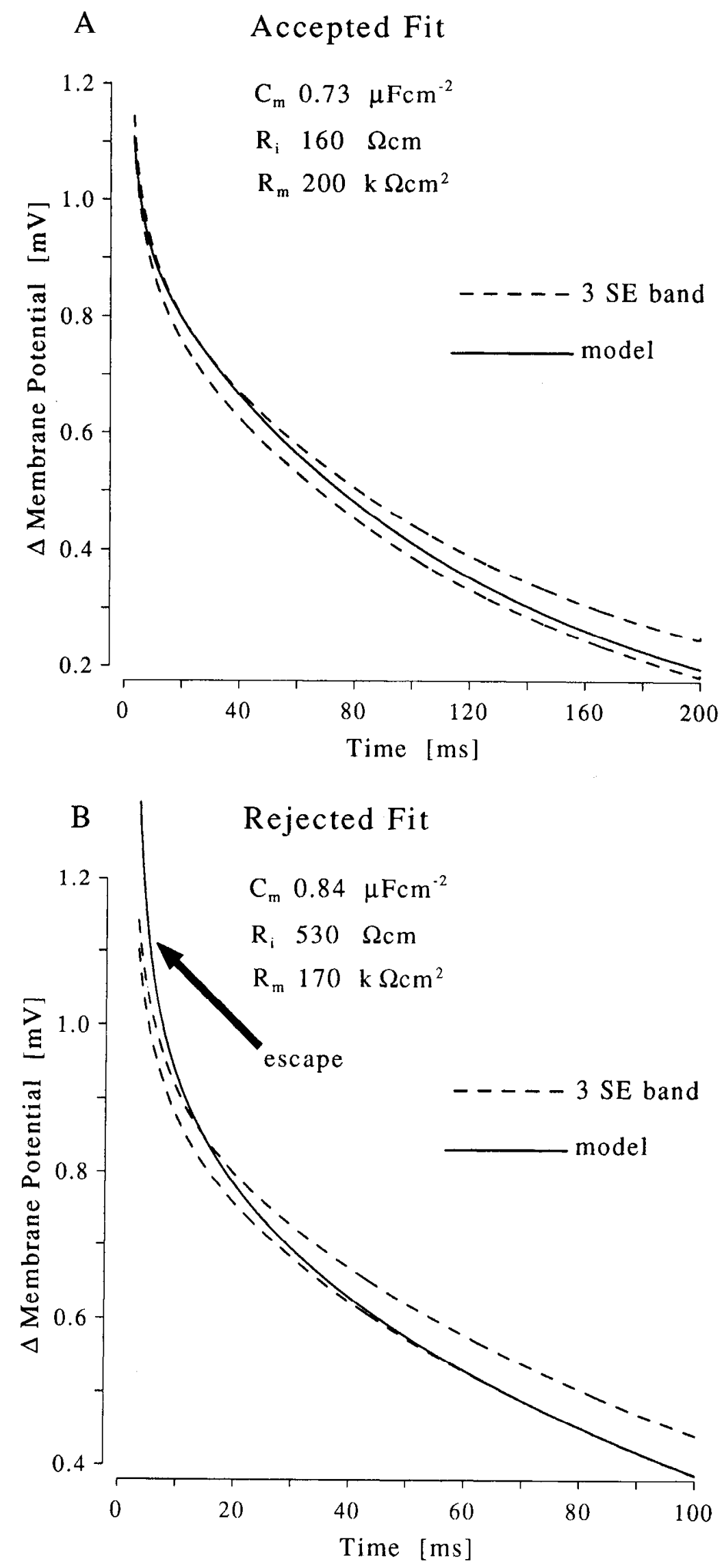

Figure 7. Fit acceptance and fit rejection by the confidence band test. CA3_32's grand average response $\sim 95 \%$ ( $3 \mathrm{SE}$ ) confidence band is shown (without the grand average mean waveform itself) as the dashed lines in both panels. $A$, Fit that is only just accepted (parameters as listed): the fit waveform (solid line) just touches the top edge of the $3 \mathrm{SE}$ band around $t=30 \mathrm{msec} . B$, Fit with $R_{i}$ too high (solid line) escapes from the 3 SE band at times before about $15 \mathrm{msec}$ (arrow). Different time axis from $A$. 


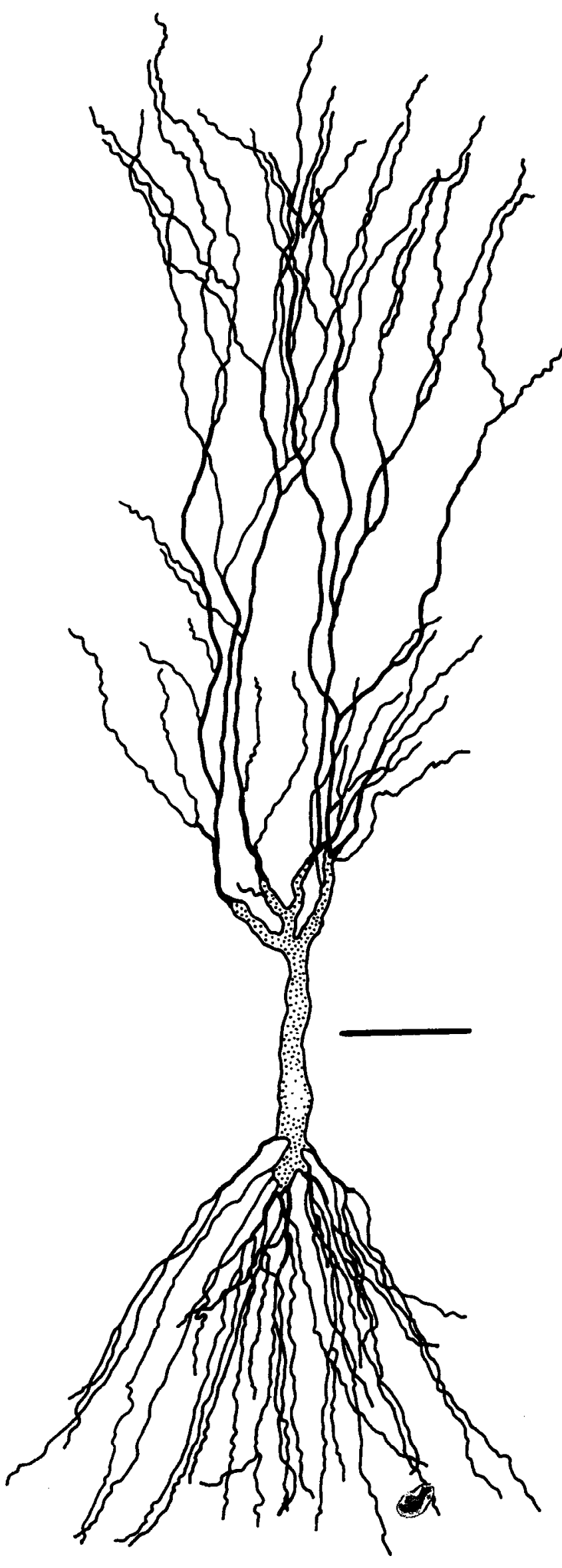

ditioned with respect to $g_{\text {shun }}$ : small changes in shunt result in enormous changes in $R_{m}$, in some cases up to implausibly high levels. This nonuniqueness is of the core electrotonic model variety, since the morphology is fixed. The different models yield different predicted PSPs, particularly at late times if the shunt is removed.

\section{Errors from omitting the axons}

The effects on the optimal fit parameters of omitting the axons from the models are summarized in Table 6. Some of the errors are worth taking seriously: they seem to be worst for short, thick proximal axon segments, but are almost independent of total axon area; this suggests that modeling errors due to axonal amputations or incomplete fills of very distal axonal segments are unlikely to be significant.

\section{Steady-state passive electrotonic structure}

The steady-state electrical geometry of the cells is summarized in Table 7, using the zero shunt fit parameters. Because of load effects and end effects (e.g., Rall, 1959, 1977; Jack ct al., 1975, chapter 7; Holmes, 1986a), electrotonic length can be misleading. The mean basal/oblique electrotonic lengths are about 0.2 space constants, whereas the apical tufts terminate at about 0.5 $\lambda$. The steady-state efficacies (voltage at soma in response to current at dendritic tip $\div$ voltage at soma in response to same current at soma) show even more electrical compactness: no average efficacy is less than $81 \%$, and the basal/oblique average efficacies are over $95 \%$, even for the electrotonically "longest" models. Because of reciprocity (Rall and Segev, 1985; Major et al., 1993b), the steady-state dendrite-to-soma efficacy is the same as the steady-state soma-to-dendrite voltage-clamp efficacy (dendritic voltage in response to somatic current $\div$ somatic voltage in response to somatic current). All of these cells would be extremely well clamped for steady-state commands. This is not the case for fast current or voltage transients, however.

\section{Simulations of transient synaptic inputs}

Despite the steady-state electrical compactness of the cells, their dendrites can still produce substantial cable filtering of fast current or voltage transients. This is illustrated in Figure 12, using CA3332's optimal model (omitting the axon). The inputs consisted of double exponential currents with kinetics based on $\alpha$-amino-3-hydroxy-5-methyl-4-isoxazole propionate (AMPA)/ kainate receptor responses in brief $(1 \mathrm{msec})$ glutamate pulse experiments on outside-out patches from $\mathrm{CA} 3$ cells at room temperature (Colquhoun et al., 1992). A total charge of $0.1 \mathrm{pC}$ was injected into one of three locations (arrowheads in Fig. 10, details in Fig. 12 caption): (1) the soma, (2) halfway along the apical oblique segment, and (3) halfway along the apical tuft segment, with median soma-to-tip morphotonic distances for their class. The onset time constant of the current was $0.2 \mathrm{msec}$, and the offset time constant $2.5 \mathrm{msec}$, giving a $20-80 \%$ rise time of 0.19 msec. A median basal input (not shown) gave similar somatic PSPs to the apical oblique input.

The PSPs at the soma are shown in Figure 12 $\mathrm{A}$, for the optimal model (black lines), and also for a model with the same param-

Figure 8. Camera lucida reconstruction of dendritic arbor of CA3_33. Scale bar, $50 \mu \mathrm{m}$. 


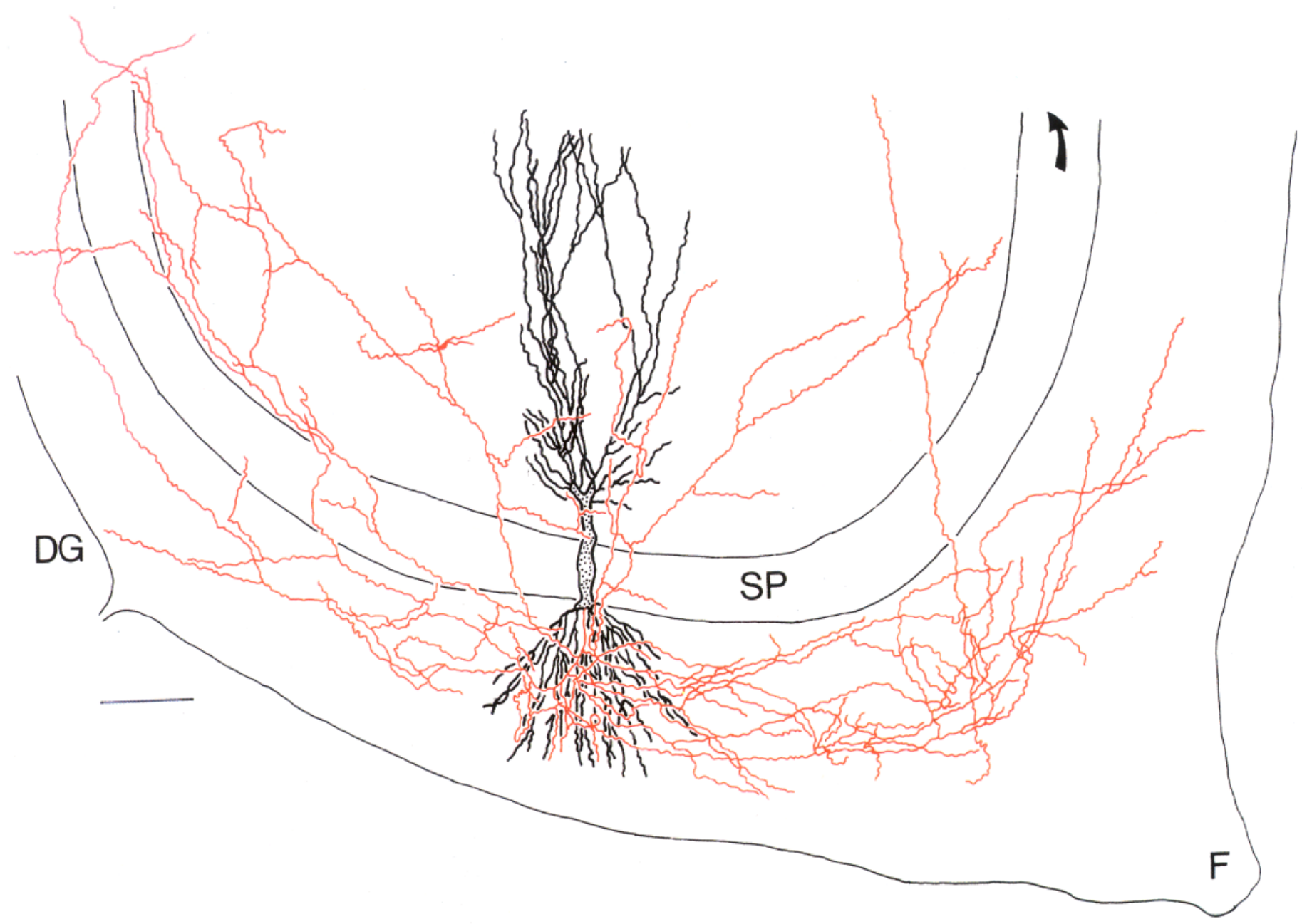

Figure 9. Camera lucida reconstruction of dendritic and axonal arbors of CA3_32. Red, axon; black, dendrite. DG, dentate gyrus; SP, stratum pyramidale; $F$, fornix. Arrow points toward CA1. Scale bar, $100 \mu \mathrm{m}$.

eters except for an additional $10 \mathrm{nS}$ shunt at the soma (to mimic a sharp electrode recording). It can be seen that there is a wide range of rise times, and that the peak amplitudes and early rising phases of the waveforms are not severely affected by the shunt. Apical tuft (perforant path) inputs' rising phases are slowed several times more than are those of apical oblique (and basal) inputs, which are predominantly from CA3 collateral axons. Mossy fiber PSPs are faster still (only slightly slower than the somatic input).

The effects of dendritic cables on the voltage-clamp currents in response to these inputs are shown in Figure 12B. With perfect somatic voltage clamp the apical oblique and apical tuft inputs give rise to clamp currents that are substantially slowed and attenuated compared with the true (or somatic) input current. Any series resistance would cause further smoothing and attenuation. The relative peak efficacy under voltage clamp is worse than under voltage recording, both with and without the shunt.

\section{Discussion}

\section{Principal findings}

This article describes a method for deriving passive cable models based on (1) combined short pulse responses and high-quality morphology from tight seal whole-cell recorded neurons, and
(2) an objective means of fit rejection, based on noise controls and SE bands around the averaged response, to establish confidence limits for model parameters. The study was conducted on CA3 pyramidal neurons in in vitro hippocampal slices taken from 19-21-d-old rats.

The main result is that, in this class of cell, with our morphological measurements, $C_{m}$ is about $0.7-0.8 \mu \mathrm{Fcm}^{-2}$, and $R_{i}$ is about $170-340 \Omega \mathrm{cm} . R_{m}$, after washout of sag, is around 120 $200 \mathrm{k} \Omega \mathrm{cm}^{2}$. The value for $C_{m}$ is close to what has been measured directly for a number of cell and membrane types (see introductory remarks). Also, in a recent study of spherical isolated rat pituitary nerve terminals (Rosenboom and Lindau, personal communication), $C_{m}$ has been measured to be $0.76 \pm 0.05$ $\mu \mathrm{Fcm}^{-2}$, remarkably close to the values we have found. It is not safe, therefore, to assume that $C_{m}$ is $1 \mu \mathrm{Fcm}^{-2}$, as is commonly done. The value of $R_{i}$ is severalfold higher than that expected from consideration of the ionic composition of the intracellular fluid (e.g., Clements and Redman, 1989), and that measured by the tip-resistance change method (Schanne, 1969) in mononeurons (Barrett and Crill, 1974). High $R_{i}$ values have been found in a number of studies to date (e.g., Shelton, 1985; Stratford et al., 1989; Fromherz and Vetter, 1992; Major et al., 1993a; Rapp et al., 1993; Thurbon et al., 1994). 

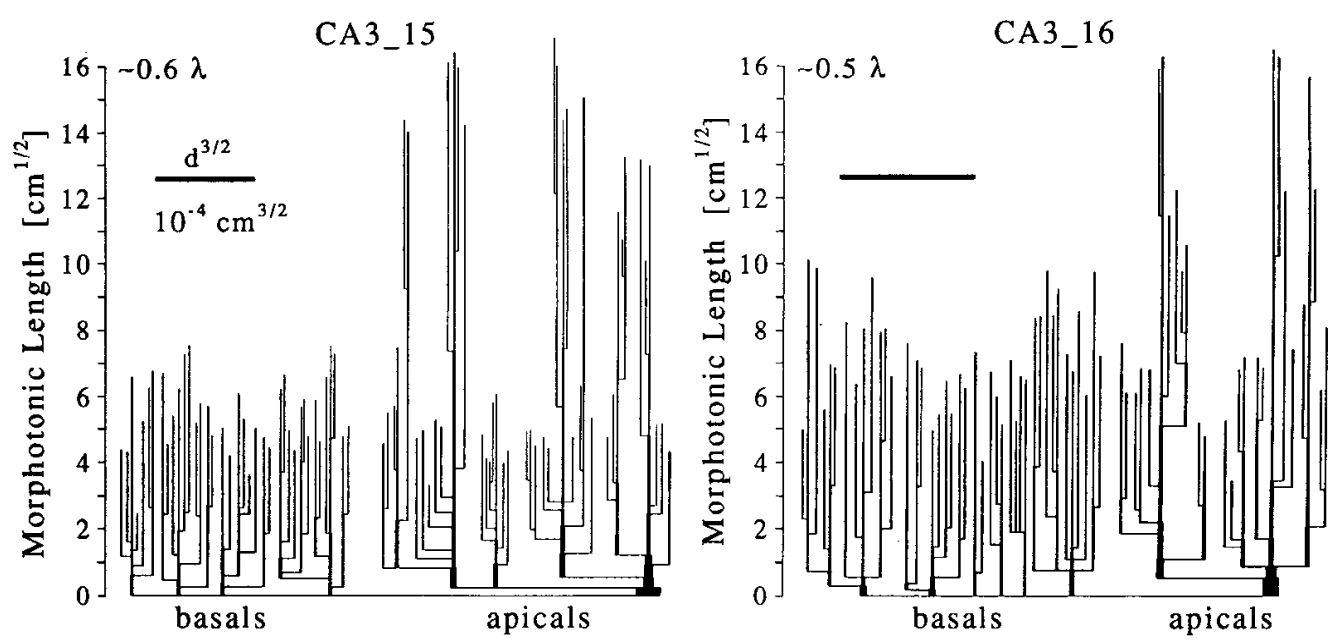

CA3_32
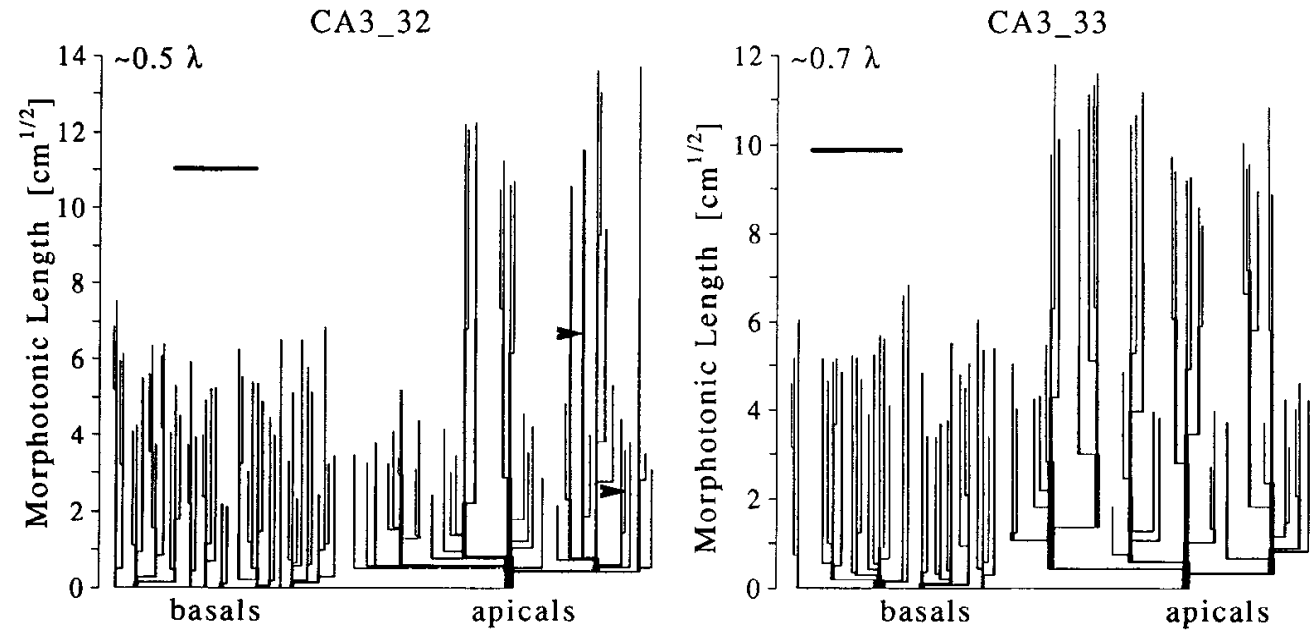

Figure 10. Morphotonic dendrograms of the four cells. A segment $j$ 's morphotonic (or relative electrotonic) length $l_{M}$ is defined as $2 l_{j} / d_{j}^{1 / 2}$, where $l_{i}$ and $d_{i}$ are its physical length and diameter (after spine incorporation). In these dendrograms, vertical lines represent segments; horizontal lines indicate branching. The length of a vertical line is proportional to $l_{3,}$ of that segment. The thickness is proportional to $d_{j}^{3 / 2}$, which is the morphological component of $g_{x_{1}}=(\pi / 2)\left(R_{m} R_{i}\right)^{-1 / 2} d_{j}^{3 / 2}$, the input conductance of that cylindrical segment, infinitely extended. The $g_{\infty}$, of each segment is an important core electrotonic model parameter in the analytical solution for that cell. The actual electrotonic length scaling for the optimal fit models (see Fig. $11)$ is indicated by the number of space constants $\left(\lambda\right.$, shown at the top of each ordinate) corresponding approximately to the upper axis limit of $l_{M}$, the morphotonic distance from the soma. For example, in the casc of CA3 $15, l_{\mathrm{M}}=16 \mathrm{~cm}^{1 / 5}$ is equal to about 0.6 space constants in the optimal fit model. Arrowheads in CA3_32 panel indicate input sites for simulations of transients (Fig. 12). Horizontal scale bars $\left(=10^{-4} \mathrm{~cm}^{3 / 2}\right)$ apply to the vertical line thicknesses $\left(d_{i}^{3 / 2}\right)$.

The entire range of electrical parameters consistent with the experimental data leads to very short electrotonic lengths for these cells' dendrites, with the basal/oblique dendrites being about 0.2 space constants and the apical trunk + tufts being about 0.5 space constants long. For constant or slow inputs, these structures are very compact electrically: the average steady-state tip-soma efficacies are greater than $95 \%$ for the basal/oblique dendrites, and greater than $81 \%$ for the apical tufts. Despite this, with fast inputs, the dendritic trees are still capable of generating a wide range of PSP shapes, and can heavily slow and attenuate postsynaptic clamp currents (for more detailed discussions, see Jonas et al., 1993; Major, 1993; Spruston et al., 1993).

The $R_{m}$ and $R_{i}$ values we have found may change with age and temperature, and the kind of preparation. Most ionic conductances have a $Q_{10}$ of between 1.3 and 1.6 (e.g., Hille, 1992, p 329). Our experiments were performed at room temperature (20-24 $4^{\circ} \mathrm{C}$; at $35^{\circ} \mathrm{C}$, commonly used for slice experiments, $R_{m}$ may therefore be lower, perhaps by a factor of $1.3-2$. The $Q_{10}$ for aqueous diffusion is about 1.3 (Hille, 1992), so $R_{i}$ may also be reduced by a factor of up to 1.5 at $35^{\circ} \mathrm{C}$. Rat body temperature is about $40^{\circ} \mathrm{C}$, so the decreases may be more pronounced in vivo. In addition, simulations have shown that effective $R_{m}$ can be substantially reduced by the background synaptic conductances (e.g., Bernander et al., 1991; Rapp et al., 1992) that are likely to occur in vivo.

\section{Fit rejection}

Any data-fitting exercise should include an attempt to estimate confidence intervals for the model parameters, as well as simply finding a single optimum fit (there may be multiple indistinguishably good optima). In order to do this, an objective test is required for fit rejection; simply comparing waveforms by eye is inadequate. In most intracellular recordings (and many other 


\begin{tabular}{|c|c|c|c|c|c|}
\hline & CA3_15 & CA3_16 & CA3_32 & CA3_33 & Mean $\pm \mathrm{SD}^{a}$ \\
\hline \multicolumn{6}{|l|}{ Total number of } \\
\hline Dendritic segments & 169 & 137 & 159 & 142 & $152 \pm 13$ \\
\hline Axonal segments & 109 & 193 & 207 & 130 & $160 \pm 41$ \\
\hline Dendritic spines & 32,400 & 28,700 & 34,900 & 2,600 & $32,200 \pm 200$ \\
\hline Boutons & 4000 & 7100 & 5900 & 4400 & $5400 \pm 1200$ \\
\hline \multicolumn{6}{|c|}{ Average density (after wiggle $\left.{ }^{h}\right)\left(\mu \mathrm{m}^{-1}\right)$} \\
\hline Dendritic spines & 2.7 & 2.5 & 3.1 & 3.3 & $2.9 \pm 0.3$ \\
\hline Boutons & 0.27 & 0.30 & 0.26 & 0.25 & $0.27 \pm 0.02$ \\
\hline \multicolumn{6}{|c|}{ Terminal segment diameters before spines incorporated $(\mu \mathrm{m})$} \\
\hline Basal & $0.7 \pm 0.1$ & $0.6 \pm 0.1^{2}$ & $0.7 \pm 0.1$ & $0.7 \pm 0.1$ & $0.7 \pm 0.0$ \\
\hline Apical oblique & $0.6 \pm 0.2$ & $0.6 \pm 0.1$ & $0.7 \pm 0.2$ & $0.7 \pm 0.1$ & $0.7 \pm 0.0$ \\
\hline Apical tuft & $0.6 \pm 0.1$ & $0.6 \pm 0.1$ & $0.8 \pm 0.2$ & $0.6 \pm 0.1$ & $0.6 \pm 0.1$ \\
\hline \multicolumn{6}{|c|}{ Terminal segment diameters after spines or boutons incorporated ${ }^{d}(\mu \mathrm{m})$} \\
\hline Basal & $0.9 \pm 0.2$ & $0.8 \pm 0.1$ & $0.9 \pm 0.2$ & $0.9 \pm 0.1$ & $0.9 \pm 0.0$ \\
\hline Apical oblique & $0.8 \pm 0.2$ & $0.7 \pm 0.1$ & $0.9 \pm 0.2$ & $0.9 \pm 0.2$ & $0.8 \pm 0.1$ \\
\hline Apical tuft & $0.7 \pm 0.1$ & $0.7 \pm 0.1$ & $0.9 \pm 0.3$ & $0.8 \pm 0.1$ & $0.8 \pm 0.1$ \\
\hline Axon & $0.2 \pm 0.1$ & $0.2 \pm 0.1$ & $0.2 \pm 0.1$ & $0.2 \pm 0.1$ & $0.2 \pm 0.0$ \\
\hline \multicolumn{6}{|c|}{ Soma-to-tip morphotonic ${ }^{e}$ distances (spines incorporated) $\left(\mathrm{cm}^{1 / 2}\right)$} \\
\hline Basal & $5.5 \pm 1.1$ & $7.1 \pm 1.5$ & $4.9 \pm 1.4$ & $4.9 \pm 0.9$ & $5.6 \pm 0.9$ \\
\hline Apical oblique & $5.0 \pm 0.8$ & $6.4 \pm 1.2$ & $3.7 \pm 0.8$ & $4.0 \pm 0.8$ & $4.8 \pm 1.1$ \\
\hline Apical tuft & $14.1 \pm 1.9$ & $13.1 \pm 2.7$ & $11.6 \pm 1.3$ & $10.0 \pm 1.0$ & $12.2 \pm 1.6$ \\
\hline \multicolumn{6}{|c|}{ Sum of terminal segment (diameter) ${ }^{3 / 2}$ values (spines incorporated) $\left(\mu \mathrm{m}^{3 / 2}\right)$} \\
\hline Basal & 31.9 & 27.2 & 36.0 & 26.8 & $30.5 \pm 3.8$ \\
\hline Apical oblique & 21.4 & 10.2 & 22.0 & 15.3 & $17.2 \pm 4.8$ \\
\hline Apical tuft & 10.9 & 7.3 & 12.3 & 15.0 & $11.4 \pm 2.8$ \\
\hline \multicolumn{6}{|l|}{ Surface area $\left(\mu \mathrm{m}^{2}\right)$} \\
\hline Soma & 2800 & 2000 & 3000 & 1500 & $2300 \pm 600$ \\
\hline Total dendritic & 59,000 & 50,300 & 63,500 & 57,500 & $57,600 \pm 4700$ \\
\hline Total axonal & 19,000 & 26,200 & 24,800 & 20,300 & $22,600 \pm 3000$ \\
\hline Total cell & 80,800 & 78,500 & 91,300 & 79,300 & $82,500 \pm 5200$ \\
\hline
\end{tabular}

${ }^{a}$ Mean \pm SD of figures in preceding four columns. Where preceding columns' data are of the form $\bar{x} \pm \sigma_{r}$, the $\sigma_{x}$ values are ignored.

"Lengths are multiplied by wiggle factor (see Experimental methods). Average density obtained by dividing total number of spines by total dendritic length (not shown).

- Figures for each cell of form $\bar{x} \pm \sigma$ are (unweighted) average \pm (within-cell) standard deviation, over all terminals of the specified class. Note that diameter figures are quoted to one decimal place only.

"Spine collapse procedure described in Experimental methods.

' Morphotonic length of segment $j$ is $l_{: t_{j}}=2 l / \sqrt{d_{j}}$, where $I_{j}$ and $d_{j}$ are the segment length and diameter, respectively. For a given tip, the soma-to-tip morphotonic distance is the sum of the $l_{M}$, values for all segments $j$ on the direct path from the soma to that tip.

kinds of data), the noise is not independent over time, as most conventional statistical tests assume.

One way around this problem is to compare fit residuals to control noise, and to assess how likely it is that the observed deviation between a given model response and the target data could have occurred by chance. It is not necessary to know anything about the model: its response waveform is all that is required. In other words, such techniques are model-free and are fairly general in their applicability; for example, they could be used to accept or reject multi-cxponential fits. Two methods are suggested above, one based on rejecting fits that escape from $95 \%$ confidence bands around the target waveform, the other on rejecting fits whose WSD or weighted root mean squared deviation from the target is greater than $95 \%$ of control noise WSDs. The confidence band test is more sensitive to large, brief deviations between model response and data; the WSD test is more sensitive to smaller, longer-lasting deviations. In this study, we have reported results using the former method, because it yielded the best-constrained ranges for $R_{i}$ and $C_{m}$, the parameters of greatest interest.

An alternative approach would have been the use of $\chi^{2}$ tests based on the inverse covariance matrix (Diggle, 1990, Appendix B). For these, it is necessary to compute from single sweeps the experimental response's covariance matrix (i.e., as well as the variance of the response at all times $t$, the covariances between the response at $t$ and all other times $t^{\prime}$ must also be found). The covariances are then converted into standard covariances (of the mean response from each $t$ to each $t^{\prime}$ ) by dividing by the weighted number of sweeps in the grand average. The standard variance (i.e., the SE squared) is just the special case when $t=$ $t^{\prime}$. The standard covariance matrix must then be inverted, before being premultiplied by the row vector and postmultiplied by the column vector of residuals, to give the goodness of fit measure. If further tests confirmed that the responses at each time 

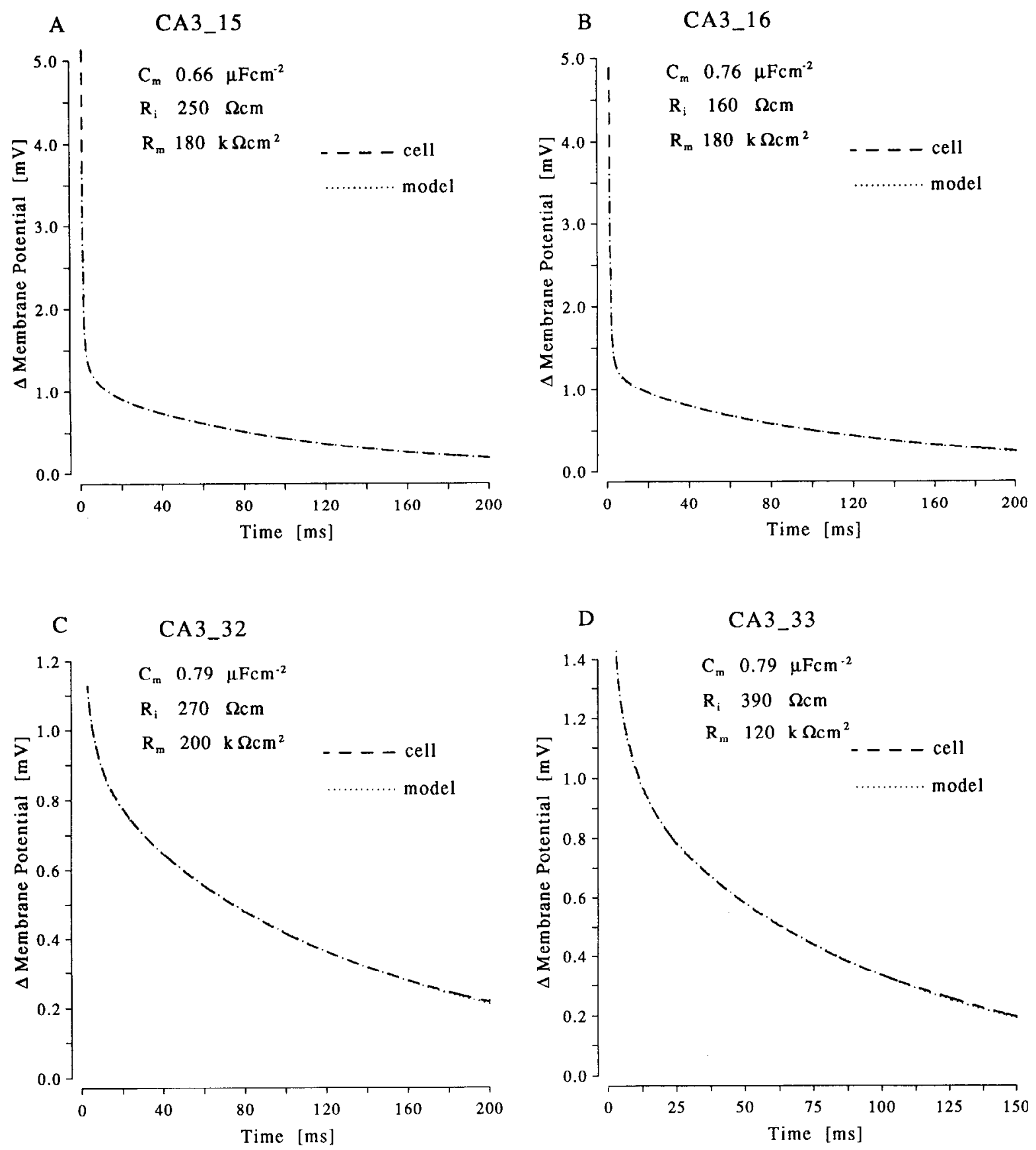

Figure 11. Optimal fits of all four cells. First two models include most likely pipette $(20 \mathrm{pF}, 10 \mathrm{M} \Omega)$, via which current is injected and response is recorded. Real cells' grand average target responses to short current pulses are dashed lines; optimal model responses to same current are dotted lines. Lines superimpose so well that they appear to be one dot-dashed line. Note different voltage axes. Optimal electrical parameters are listed in each panel.

point were (approximately) normally distributed about their mean, this quantity would have, asymptotically, a $\chi_{n}^{2}$ distribution, where $n$, the degrees of freedom, is (number of points in fit interval) - (number of free parameters). In such a case, there would be no need for independent noise controls. If the scatter at each time point was not normally distributed, the same procedure would have to be applied to independent noise controls to estimate significance levels. Since our fit intervals encompass on the order of 2000 points typically, a $(2000 \times 2000)$ matrix inversion would be required. The method would suffer from dilution problems (as when there are no temporal correlations in the noise); for example, a poor fit at early times can be swamped in the goodness of fit measure by an extremely good fit over the rest of the interval. There may be some simpler analytical approaches, exploiting the apparently straightforward exponential decay of the correlations.

All the tests mentioned could probably be made less sensitive to dilution, for example, by judicious use of (1) weighting profiles (e.g., multiply the error at each time by the magnitude of the model or experimental response, so all residuals end up being weighted by the signal-to-noise ratio, which decreases over the fit interval), and (2) nonuniform sample intervals in the grand 
Table 3. Approximate input resistances and time constants, from short pulse responses

\begin{tabular}{|c|c|c|c|c|}
\hline \multirow[b]{2}{*}{ Cell } & \multicolumn{2}{|c|}{ "Initial"' $a$} & \multicolumn{2}{|c|}{ Postwashout $^{b}$} \\
\hline & $\begin{array}{l}\tau_{0}{ }^{c} \\
\text { (msec) }\end{array}$ & $\begin{array}{l}R_{N}^{d} \\
(\mathrm{M} \Omega)\end{array}$ & $\begin{array}{l}\tau_{0}{ }^{\circ} \\
\text { (msec) }\end{array}$ & $\begin{array}{l}R_{N}{ }^{d} \\
(\mathrm{M} \Omega)\end{array}$ \\
\hline CA3_15 & 75 & 180 & 120 & 260 \\
\hline CA3_16 & 140 & 280 & 140 & 310 \\
\hline CA3_32 & 94 & 170 & 150 & 260 \\
\hline CA3_33 & 63 & 140 & 93 & 200 \\
\hline Mean & 93 & 190 & 130 & 260 \\
\hline
\end{tabular}

Values are quoted to two significant figures.

"First subaverage of 100 sweeps; some washout already.

"Grand average of linearly scaling, stable subaverages showing no sag; see Modeling methods.

Apparent slowest time constant estimated by linear regression using logarithmic voltage axis, over following intervals (times in milliseconds from pulse end): initial responses, 50-100 (except CA3_16, 50-200); postwashout responses, 100-200 (except CA3_33, 100-150). Time constants from initial subaverages particularly unreliable due to contaminating spontaneous synaptic noise.

${ }^{d}$ Exponential amplitudes and time constants estimated from short pulse responses by peeling (Rall, 1969a). Approximate input resistances $R_{x}$ were calculated using Equation 4 (see Modeling methods). Early components (with $n$ greater than some $n_{\max }$, ranging from 3 to 5 ) having time constants $\tau_{u} \leq 1.5 \tau_{\text {pip }}$ were excluded, where $\tau_{\text {pip }}$ was the slowest expected value of the pipette time constant, on the basis of highest series resistance $\times$ largest estimate of pipette capacitance.

average (but not the unfiltered single sweeps): the sample interval could be increased with time from the pulse end, so late times make a smaller contribution to the goodness of fit measure. This would have the advantage, with the covariance matrix method, of reducing the size of the matrix. Because of the heavy filtering at late times, comparatively little information about the underlying response would be lost.

Such approaches would lead to a narrowing of the confidence intervals for $R_{i}$ and $C_{m}$ for a given cell, but would have little effect upon intercell variation. In this study, since the inter- and intracell ranges for the parameters are similar, there would not

\begin{tabular}{|c|c|c|c|c|c|c|}
\hline Cell & $\begin{array}{l}g_{\text {shunt }} \\
\text { (nS) }\end{array}$ & $\begin{array}{l}C_{m} \\
\left(\mu \mathrm{Fcm}^{-2}\right)\end{array}$ & $\begin{array}{l}R_{i} \\
(\Omega \mathrm{cm})\end{array}$ & $\begin{array}{l}R_{m} \\
\left(\mathrm{k} \Omega \mathrm{cm}^{2}\right)\end{array}$ & WSD & $\begin{array}{l}\Lambda^{b} \\
\left(\mathrm{~cm}^{1 / 2}\right)\end{array}$ \\
\hline \multirow[t]{3}{*}{ CA3_15 } & 1 & 0.63 & 230 & 260 & 0.13 & 33 \\
\hline & 2 & 0.60 & 216 & 440 & 0.17 & 45 \\
\hline & 3 & 0.54 & 224 & 2000 & 0.49 & 94 \\
\hline \multirow[t]{3}{*}{ CA3_16 } & 1 & 0.73 & 150 & 280 & 0.28 & 43 \\
\hline & 2 & 0.70 & 150 & 650 & 0.18 & 65 \\
\hline & 3 & 0.64 & 170 & 2000 & 2.96 & 111 \\
\hline \multirow[t]{3}{*}{ CA3_32 } & 1 & 0.77 & 260 & 280 & 0.25 & 33 \\
\hline & 2 & 0.75 & 240 & 460 & 0.18 & 44 \\
\hline & 3 & 0.73 & 230 & 1200 & 0.14 & 74 \\
\hline \multirow[t]{5}{*}{ CA3_33 } & 1 & 0.76 & 390 & 150 & 0.51 & 20 \\
\hline & 2 & 0.74 & 380 & 210 & 0.50 & 23 \\
\hline & 3 & 0.71 & 380 & 330 & 0.50 & 30 \\
\hline & 4 & 0.69 & 370 & 740 & 0.51 & 45 \\
\hline & 5 & 0.66 & 330 & $1.8 \times 10^{6}$ & 1.2 & 2300 \\
\hline
\end{tabular}

Values are quoted to two significant figures only.

"All accepted by $\sim 95 \%$ confidence band test.

b Morphoelectric factor $\left(R_{m} / R_{\ell}\right)^{\prime \prime}$.

- Models include most likely pipette (10 M, $20 \mathrm{pF})$.

be any significant advantage compared to the cruder confidence band method described above.

\section{Potential sources of error}

Random errors due to spontaneous synaptic noise have been minimized by the use of neurotransmitter blockers. Efforts to improve the stability of recordings could further reduce noise. The statistical tests described above have, we hope, provided fairly conservative $95 \%$ confidence intervals for all of the electrical parameters. Systematic errors due to pipette artifacts have either been avoided, or the pipettes have been included in the models. Sag, linear scaling, and stability have been explicitly

Table 4. Summary of parameters obtained from direct fits between model and experimental responses with $g_{\text {shumt }}$ fixed to zero

\begin{tabular}{|c|c|c|c|c|c|c|c|c|c|c|}
\hline \multirow[b]{2}{*}{ Cell } & \multicolumn{2}{|c|}{$\begin{array}{l}C_{m} \\
\left(\mu \mathrm{Fcm}^{-2}\right)\end{array}$} & \multicolumn{2}{|c|}{$\begin{array}{l}R_{i} \\
(\Omega \mathrm{cm})\end{array}$} & \multicolumn{2}{|c|}{$\begin{array}{l}R_{m} \\
\left(\mathrm{k} \Omega \mathrm{cm}^{2}\right)\end{array}$} & \multicolumn{2}{|c|}{ Fit $\mathrm{WSD}^{a}$} & \multicolumn{2}{|c|}{$\begin{array}{l}\Lambda^{b} \\
\left(\mathrm{~cm}^{1 / 2}\right) \\
\end{array}$} \\
\hline & Best & Range & Best & Range & Best & Range & Best & Range & Best & Range \\
\hline $\mathrm{CA}^{3} \_15^{\circ}$ & 0.66 & $0.56-0.77$ & 250 & $100-400$ & 180 & $210-160$ & 0.13 & $0.13-2.6$ & 27 & $42-21$ \\
\hline CA3_16 & 0.76 & $0.73-0.81$ & 160 & $110-210$ & 180 & $220-160$ & 0.35 & $0.28-3.0$ & 34 & $42-29$ \\
\hline $\mathrm{CA} 3 \_32^{d}$ & 0.79 & $0.73-0.83$ & 270 & $160-320$ & 200 & $220-180$ & 0.30 & $0.30-2.4$ & 27 & $35-25$ \\
\hline $\mathrm{CA} 3 \_33^{d}$ & 0.79 & $0.74-0.81$ & 390 & $300-410$ & 120 & $130-110$ & 0.54 & $0.54-2.1$ & 17 & $20-17$ \\
\hline Mean & 0.75 & $0.69-0.81$ & 270 & $170-340$ & 170 & $200-150$ & - & - & 26 & $35-23$ \\
\hline
\end{tabular}

Data are fits to grand averages, over intervals specified in Table 1 . Acceptable ranges were determined by systematic exploration of parameter space and noting most extreme values where fit waveform just inside $\sim 95 \%$ confidence band of target data (see Table 1). Ranges in mean row are averages of the values in the column above them. Fits with higher $C_{m}$ values tended to be associated with higher $R_{i}$ values and lower $R_{m}$ and hence lower $\Lambda$ values, so $R_{m}$ and $\Lambda$ ranges are written as highest-lowest. Corresponding extreme parameters are not necessarily from the same fit. Values are quoted to two significant figures only.

"Weighted standard deviation between target and model transients, over fit interval (= root mean squared deviation, with squared error at each time point normalized by $S^{2}$ of target data)

"Morphoelectric factor $\left(R_{m} / R_{i}\right)^{\prime \prime}$.

" "Best" models have most likely pipette (10 M, $20 \mathrm{pF}$ ); also tested: $5 \mathrm{M} \Omega, 10 \mathrm{pF}$ (best plausible pipette); $15 \mathrm{M} \Omega, 20$ $\mathrm{pF}$ (worst plausible pipette). SEs of each data point were computed from subaverages, not individual sweeps. Confidence band was filtered with $\sigma=0.2 t$ variable gaussian filter.

${ }^{a}$ Pipette not included in model. Standard errors were computed from all single sweeps used for grand average. 


\begin{tabular}{|c|c|c|c|c|c|c|}
\hline \multirow[b]{2}{*}{ Cell } & \multirow[b]{2}{*}{$\begin{array}{l}C_{m} \\
(\%)\end{array}$} & \multirow[b]{2}{*}{$\begin{array}{l}R_{i} \\
(\%)\end{array}$} & \multirow[b]{2}{*}{$\begin{array}{l}R_{m} \\
(\%)\end{array}$} & \multicolumn{2}{|c|}{$\begin{array}{l}\text { Most proximal } \\
\text { axon segment }\end{array}$} & \multirow{2}{*}{$\begin{array}{l}\text { Total } \\
\text { area } \\
\div \\
\text { den- } \\
\text { dritic } \\
\text { area }\end{array}$} \\
\hline & & & & $\begin{array}{l}\text { Diam- } \\
\text { eter } \\
(\mu \mathrm{m})\end{array}$ & $\begin{array}{l}\text { Length } \\
(\mu \mathrm{m})\end{array}$ & \\
\hline CA3_15 & +9 & +5 & -19 & 2.0 & 77 & 1.3 \\
\hline CA3_16 & +5 & +3 & -19 & 1.2 & 130 & 1.6 \\
\hline CA3_32 & +10 & +12 & -25 & 2.0 & 40 & 1.4 \\
\hline CA3_33 & +5 & +1 & -11 & 1.6 & 115 & 1.4 \\
\hline Mean & +7 & +5 & -18 & 1.7 & 91 & 1.4 \\
\hline
\end{tabular}

checked for, to avoid spurious curvature in grand averages (e.g., that caused by adding together responses with different final time constants), and to minimize the risk that active conductances have distorted the responses.

\section{Tissue shrinkage}

By adopting an electron microcopy-based fixation and embedding strategy, in particular by osmicating the sections prior to dehydration and by embedding in epoxy resin, it was hoped that tissue shrinkage would be kept to a minimum. However, slices shrink by about $10 \%$ during the histological procedure. With this method shrinkage generally affects the extracellular compartment of the tissue more than the intracellular. In filled cells, the reaction product may act like a kind of endoskeleton, which leads to snaking or wiggle, rather than shrinkage. In view of these considerations, no additional shrinkage factor was applied to either the lengths or the diameters. Nevertheless, considerable uncertainty remains about possible systematic errors in the morphological measures. Lengths may have been underestimated by around 10\%. Diameters could either have been decreased by shrinkage, or perversely, increased (by bunching up, as when a worm contracts). See Larkman and Mason (1990), Peters et al. (1985), West et al. (1988), and Beaulieu and Colonnier (1983) for further discussion. Typically dendritic wiggle factors were in the range of $1.1-1.15$ (i.e., about what one would expect given $10 \%$ shrinkage). Usually axons wiggled more than dendrites, probably because their courses were more erratic to begin with. The estimated $C_{m}$ of a cell will trade off with its measured surface area (see next two sections). Uniform $10 \%$ linear shrinkage would result in the measured area being only $81 \%$ of the actual area at the time of recording. To conserve total capacitance, best fit $C_{m} \times$ measured area $=$ true $C_{m} \times$ unshrunk area. If the optimum fit $C_{m}$ of the shrunk cell model was $0.75 \mu \mathrm{Fcm}^{-2}$, the true $C_{m}$ would be $0.75 \times 0.81 \approx 0.61$ $\mu \mathrm{Fcm}^{-2}$.

\section{Other morphological errors}

Diameters might have been overestimated because of diffraction, blurring, or the histological procedure. However, the mean dendritic terminal segment diameters before spine incorporation (Table 2) were about $0.6-0.7 \mu \mathrm{m}$, comparable to values measured with the electron microscope for CAl pyramidal cells (Harris and Stevens, 1989, Table 1: mean $0.56 \mu \mathrm{m}$; Harris et al., 1992, Table 2, oblique dendrite entry: day $15=0.79 \mu \mathrm{m}$, adult $=0.63 \mu \mathrm{m}$ ). The spine counts may have been in error by up to $15 \%$, and mean single spine areas may have been different from the $0.83 \mu \mathrm{m}^{2}$ assumed. To calibrate the light microscopy results, electron microscopic (EM) measures could be obtained for diameter and spines, perhaps using high-voltage EM (e.g., Wilson, 1987; Hama et al., 1989).

Equation 1 would have been reasonably accurate for dendrites, which are fairly straight apart from relatively high-frequency wiggling. Computer-aided three-dimensional reconstruction might allow more accurate estimation of axonal lengths however. The reassuringly close match between the $C_{m}$ values we have found, and more direct measures (see above), suggests

Table 7. Steady-state electrical geometry

\begin{tabular}{|c|c|c|c|c|c|c|}
\hline \multirow[b]{2}{*}{ Cell } & \multicolumn{2}{|l|}{ Basal } & \multicolumn{2}{|c|}{ Apical oblique } & \multicolumn{2}{|l|}{ Apical tuft } \\
\hline & $L^{a}$ & $\begin{array}{l}\% \\
\text { Efficacy" }\end{array}$ & $L$ & $\begin{array}{l}\% \\
\text { Efficacy }\end{array}$ & $L$ & $\begin{array}{l}\% \\
\text { Efficacy }\end{array}$ \\
\hline \multicolumn{7}{|c|}{ Optimal models } \\
\hline $\mathrm{CA} 3 \_15^{c}$ & $0.20 \pm 0.04$ & $97 \pm 1$ & $0.18 \pm 0.03$ & $97 \pm 1$ & $0.52 \pm 0.07$ & $88 \pm 3$ \\
\hline CA3_16 & $0.21 \pm 0.04$ & $97 \pm 1$ & $0.19 \pm 0.03$ & $97 \pm 1$ & $0.38 \pm 0.08$ & $92 \pm 2$ \\
\hline $\mathrm{CA} 3 \_32^{d}$ & $0.18 \pm 0.05$ & $98 \pm 1$ & $0.14 \pm 0.03$ & $97 \pm 1$ & $0.43 \pm 0.05$ & $92 \pm 2$ \\
\hline CA3_33 $3^{d}$ & $0.28 \pm 0.05$ & $96 \pm 1$ & $0.23 \pm 0.05$ & $93 \pm 1$ & $0.57 \pm 0.06$ & $84 \pm 2$ \\
\hline Average & $0.22 \pm 0.05$ & $97 \pm 1$ & $0.19 \pm 0.03$ & $96 \pm 1$ & $0.48 \pm 0.07$ & $89 \pm 2$ \\
\hline \multicolumn{7}{|c|}{ Most electrotonically "long" models } \\
\hline CA3_15 & $0.26 \pm 0.05$ & $95 \pm 2$ & $0.23 \pm 0.04$ & $95 \pm 2$ & $0.66 \pm 0.09$ & $81 \pm 4$ \\
\hline $\mathrm{CA} 3$ 3.16 & $0.25 \pm 0.05$ & $96 \pm 1$ & $0.22 \pm 0.04$ & $96 \pm 1$ & $0.46 \pm 0.09$ & $89 \pm 3$ \\
\hline
\end{tabular}

Figures for each cell are quoted as (unweighted) mean $\perp$ (within-cell standard deviation), over all terminals of specificd class. Figures in average row are (average of means) \pm (average of within-cell standard deviations).

“Soma-to-tip electrotonic distance: the soma-to-tip morphotonic distance is divided by the morphoelectric factor $\Lambda=$ $\left(R_{m,} / R_{t}\right)^{\prime ;} ;$ see Tables 2 and 4.

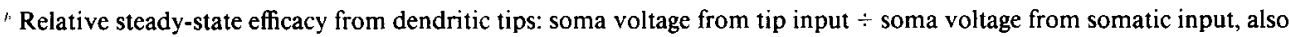
$=$ steady-state voltage-clamp efficacy; note that steady-state efficacy $=$ efficacy of time integral of transient (Rinzel and Rall, 1974).

- Most likely pipette (10 M $2,20 \mathrm{pF}$ ).

"Also approximately equal to most electrotonically long model from same cell.

- Best plausible pipette (5 M, $10 \mathrm{pF}$ ). 


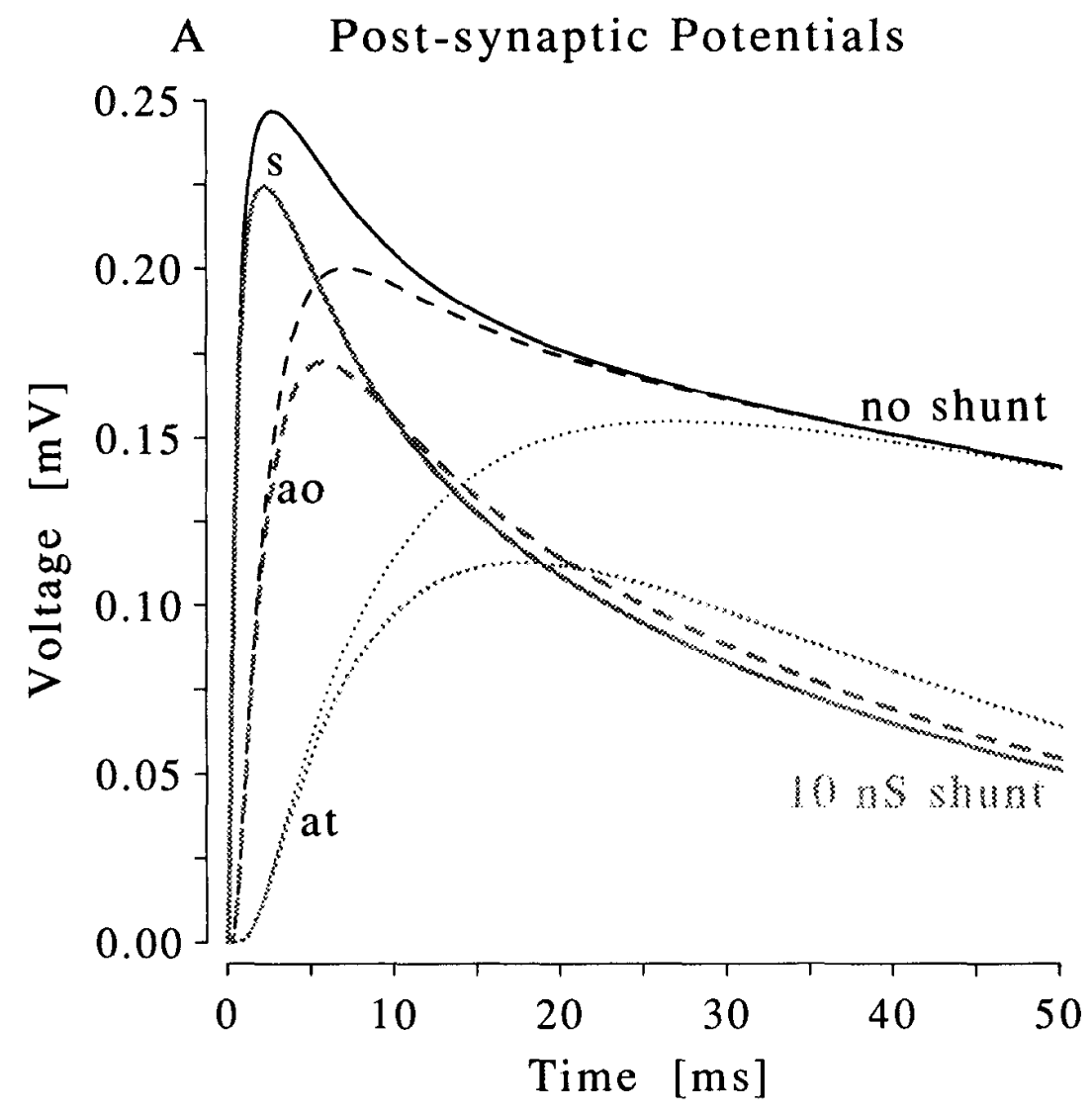

Figure 12. Simulations of transient currents into CA3_32 optimal model (axon omitted). Current parameters: double exponential time course; total charge, $0.1 \mathrm{pC}$; onset time constant, 0.2 msec; offset time constant, $2.5 \mathrm{msec}$. Three stimulus locations are used: $s$ (soma; solid lines), ao (median apical oblique: $43 \mu \mathrm{m}$ along segment 152; dashed lines), at (apical tuft: $297 \mu \mathrm{m}$ along segment 141 ; dotted lines); the latter two are arrowed in Figure 10, CA3_32 panel. Recording was from soma in all cases. $A$, Voltage recording, either with no shunt (black lines) or a $10 \mathrm{nS}$ somatic shunt (thicker gray lines). Notice the wide spread of PSP shapes, the convergence at late times for the noshunt model but not in the case of the shunt model, and the comparatively small effect of the shunt on the early parts of the responses, including the peaks of the more proximal inputs. $B$, Equivalent clamp currents under perfect somatic voltage clamp. Notice the serious distortion of the apical oblique and tuft currents, compared with the true or somatic input current (similar effects demonstrated in other studies, e.g., Fig. 7 of Rall and Segev, 1985).

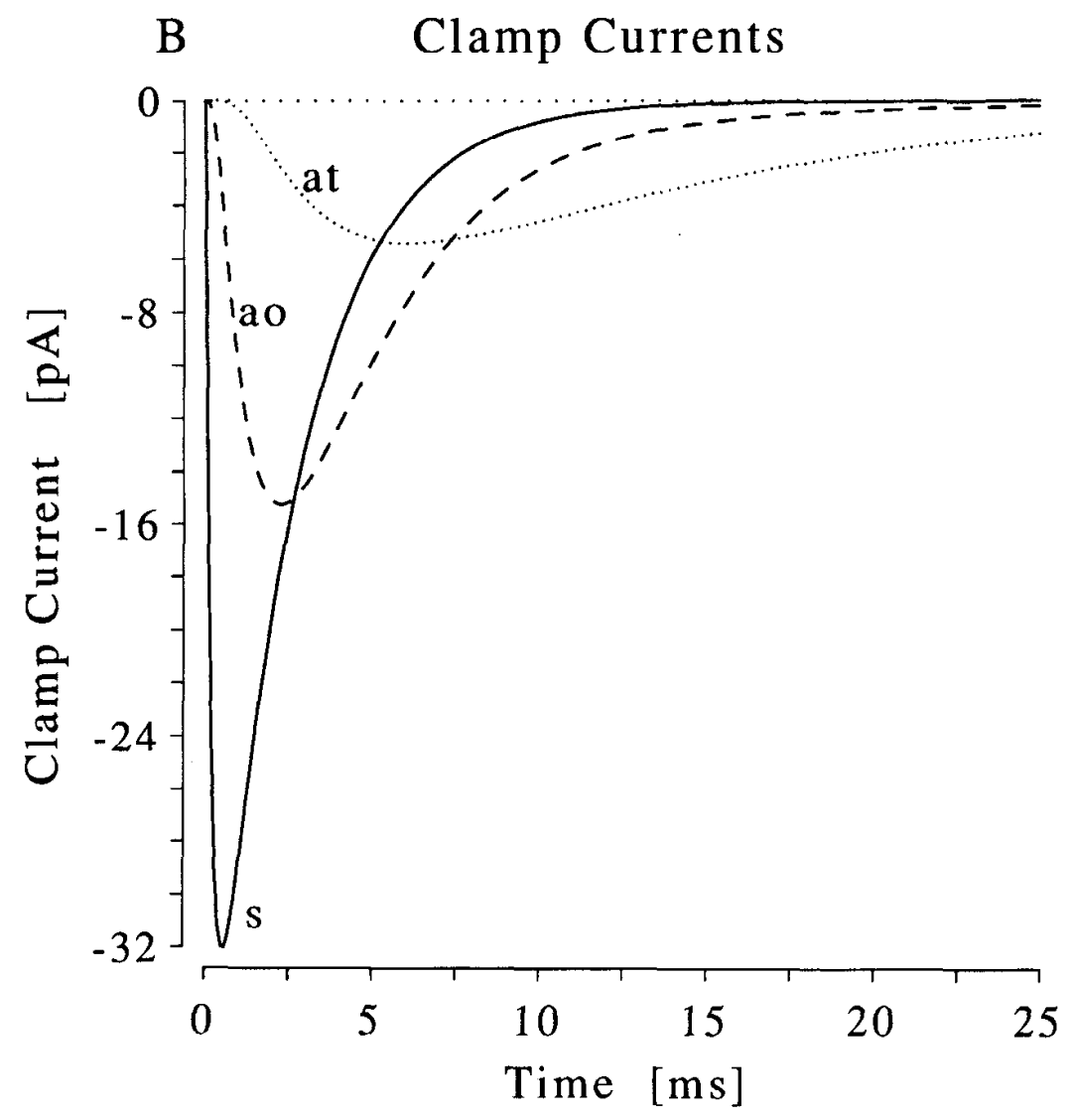


(but does not prove) that no serious errors have been made in the overall areas of these cells.

\section{Electrical parameter trade-offs with morphological errors}

There are two ways to think of a cable model with a particular branching pattern: (1) the "raw" electromorphological description, in terms of the lengths $l_{j}$ and diameters $d_{j}$ of all segments $j$, the soma diameter $d_{s}$, and the specific electrical parameters $C_{m}, R_{i}$, and $R_{m}$ (assuming no somatic shunt); and (2) the "core" electrotonic model, specified in terms of the parameters membrane time constant $\tau_{m}\left(=R_{m} C_{m}\right)$ and soma membrane conductance $g_{s m}$, and the electrotonic length $L_{j}$ and the input conductance $g_{\infty}$ (infintely extended cylinder) of each segment $j$.

It is the core electrotonic model that actually generates waveforms. If there are $N$ segments, the raw electromorphological description has $2 N+4$ parameters, but the core electrotonic model only has $2 N+2$ parameters (again, assuming no somatic shunt).

A similar duality holds for compartmental models: instead of a core electrotonic model, there is a core electrical model, composed of a branching network of actual resistances and capacitances, which is used to generate waveforms. If the membrane is uniform, and there are $N$ compartments, there are again $2 N$ +4 raw parameters, and $2 N+2$ core parameters: $C_{s}$ (soma capacitance) and $R_{s}$ (soma resistance), and for every compartment $j, R_{a x_{i}}$ (axial resistance) and $C_{j}$ (lumped membrane capacitance); the lumped membrane resistance of every dendritic compartment is then determined $\left(=C_{s} R_{s} / C_{j}\right)$.

Both cable models and compartmental models thus have the important property that a given waveform-generating core model can be obtained from an infinite number of different raw electromorphological descriptions: because there are more raw than core parameters, the raw parameters can trade off with one another and still yield the same core model, and hence the same responses (for more details, see Holmes and Rall, 1992; Major et al., 1993a; Evans et al., 1994; Major and Evans, 1994).

Consider the effects of morphological measurement errors for an aspiny neuron. Suppose that to compensate for systematic errors, all measured segment lengths are increased by a factor $F_{l}$, and all measured diameters are increased by a factor of $F_{d}$. The underlying core electrotonic model (and hence the short pulse response) is preserved by the following compensating changes in the raw specific electrical parameters: multiply $R_{i}$ by $F_{d l} / F_{l}$ and $R_{m}$ by $F_{d} F_{l}$ and divide $C_{m}$ by $F_{d} F_{l}$. The last pair of changes conserve $\tau_{m}$ and the total membrane capacitance and resistance of each segment. The first pair conserve the axial resistances, $L_{j}$ and $g_{\infty}$, of all segments $j$. (Other kinds of morphological error, such as adding or subtracting a constant from every diameter, have to be explored explicitly.)

In the case of a spiny neuron, if the dendritic spine folding factor $F_{j}$ on every dendrite (see Morphology, in Experimental methods) is increased by a factor $F_{r}$ (to approximate an increase in either single spine areas or spine density), then $F_{d}=F_{l:}^{1 / 2}$ and $F_{t}=F_{\%}$. (Note that when diameters vary this is not equivalent to multiplying all spine densities or single spine areas by the same factor.) It follows that to preserve the original core electrotonic model, $R_{i}$ is unchanged, and $R_{m}$ and $C_{m}$ are multiplied and divided, respectively, by $F_{r}$. A nonuniform change in $F_{j}$, caused for example by a uniform fractional change in spine density or single spine area, will affect $R_{i}$, but only slightly in general.

The typical dendritic terminal segment diameter $d$ (see Table
2) was $0.7 \mu \mathrm{m}$ before spine incorporation. Suppose all these diameter measures were overestimates by $0.2 \mu \mathrm{m}$, and that the mean diameter was in fact $0.5 \mu \mathrm{m}$. Assuming a spine density of $2.9 \mu \mathrm{m}^{-1}$ gives a folding factor $F$ of 2.09 with a $d$ of $0.7 \mu \mathrm{m}$, and 2.53 with a $d$ of $0.5 \mu \mathrm{m}$. After spine incorporation, the adjusted diameters are 0.9 and $0.68 \mu \mathrm{m}$, respectively; the dendritic lengths are multiplied by 1.64 when $d$ is 0.7 and by 1.86 when $d$ is only $0.5 \mu \mathrm{m}$. Overall, correcting $d=0.7$ to $d=0.5$ results in an $F_{d} \approx 0.76$ and $F_{l} \approx 1.13$, after spine incorporation. Taking these factors to hold over the entire cell (although one would expect smaller errors for the thicker nonterminal segments), to preserve the same core electrotonic model (and therefore the optimum fit waveform), $R_{i}$ would have to be multiplied by $0.76^{2} / 1.13=0.51$; that is, it would be approximately halved. Over the four cells in this study, this would result in an actual mean $R_{i}$ of about $140 \Omega \mathrm{cm}$, only about twice that commonly assumed. $R_{m}$ and $C_{m}$ would be multiplied and divided, respectively, by a factor of 0.86 ; that is, the mean $C_{m}$ would be about $0.87 \mu \mathrm{Fcm}^{-2}$.

The optimal value of $R_{i}$ is thus very sensitive to errors in diameters, since in the trade-offs it is proportional to $F_{d}^{2}$. This may go some way toward explaining the large intercell variation in $R_{i}$. It is clearly dangerous to transplant electrical parameters obtained from one study to morphology measured with different systematic errors in another study.

It should be emphasized that the adjusted model's electrotonic lengths, steady-state efficacies, and predicted PSPs and PSCs would be exactly the same as for the original model, providing the inputs were at the same relative locations (i.e., if the distance along the input segment of the injection site were multiplied by $\left.F_{l}\right)$. This is because, in terms of the core electrotonic representation, the adjusted and original electromorphological descriptions are the same model.

Extensive simulations have shown that other plausible kinds of morphological error can usually be reasonably well compensated for by changes in the specific electrical parameters when direct fitting to a particular target transient is performed. The core models and hence predicted PSP or PSC waveforms are approximately preserved, even if $R_{i}$ or $C_{m}$ are substantially different from their "true" values (see Major, 1992, chapter 4, for more discussion).

\section{Other explanations for high $\mathrm{R}_{\mathrm{i}}$}

Part of the discrepancy from the traditional value of around 70 $\Omega \mathrm{cm}$ may lie in the observation that a significant fraction of the cross-sectional area of neuronal processes can be occluded by organelles and cytoskeletal elements (e.g., Stevens et al., 1988; for general review, see Fulton, 1982). Dendritic cross sections could be elliptical or irregular, rather than circular, which would increase the effective $R_{i}$. In addition, the properties of intracellular water differ from that of pure water or water in electrolyte solutions. Self-diffusion coefficients can be two- to sevenfold lower and microviscosity may be up to five times higher in cell water than in pure water (e.g., Clegg, 1984a,b). A number of studies show reduced diffusion of ions or molecules in a variety of different cell types (e.g., Kushmeric and Podolsky, 1969; Wojcieszyn et al., 1981; Connor and Ahmed, 1984; Jacobson and Wojcieszyn, 1984; Mastro et al., 1984). See Shelton (1985) for further discussion, including a consideration of the effects of nonnegligible extracellular resistivity. The effects on $R_{i}$ of organelles, cytoskeleton, and cytomatrix, and so on, may well vary over different parts of a neuron; in this study we have not 
entertained the unpleasant possibility of nonuniform electrical parameters in the dendrites.

\section{Washout and inward rectification}

It is possible that cytoplasmic changes caused by intracellular perfusion may have affected $R_{i}$. The extra gluconate anions diffusing from the pipette into the cell could, in theory, reduce $R_{i}$ from its true value. With $\mathrm{Cs}^{+}$and/or $\mathrm{Cl}^{-}$ions in the pipette, $R$, might end up lower still, since they are more mobile than $\mathrm{K}^{+}$ and gluconate ions, respectively. The cytomatrix or cytoskeleton, and conscquently $R_{i}$, could be altered by changes in intracellular calcium concentration. The early parts of short pulse responses are sensitive to changes in $R_{i}$ and $C_{i n}$ (but not $g_{\text {shunt }}$ or $R_{m}$ : see Major et al., 1993a). Judging from the stability during washout of the short pulse responses over the interval of about $1-20 \mathrm{msec}$ from the pulse end (see Figs. $2 E, 5 B, C$ ), it is unlikely, therefore, that there were any significant changes in $R_{i}$ (or for that matter $C_{m}$ ) during the course of the recordings. Any slight boosting of the first few milliseconds of the responses is more likely to have been caused by rising pipette resistance.

In this study it was fortunate that the conductance(s) responsible for sag washed out within about 8 min of achieving the whole-cell configuration. The conductance underlying $I_{h}$ (the hyperpolarizing activating current), which contributes to sag in a number of other cell classes, is activated by cAMP (e.g., Tokimasa and Akasu, 1990; Banks et al., 1993). This intracellular messenger probably diffuses out of the cell and into the pipette. The location of the sag conductance(s) is unknown; they could be entirely in the soma, or spread through some or all of the dendrites. Assuming they are uniformly spread, one could guess that prior to washout, $\tau_{0}$ and hence $R_{m}$ (assuming no change in $C_{m}$ ) would be about half their final values. This would give a prewashout effective $R_{m}$ of around $85 \mathrm{k} \Omega \mathrm{cm}^{2}$. All electrotonic lengths would be increased by a factor of about 1.4 ; because of end effects the cells would still have a very compact steady-state electrical geometry. If all the sag conductances were in the soma, the electrotonic lengths and steady-state relative efficacies would not change with washout; they do not depend on the size of the effective somatic shunt (e.g., Appendix 3 of Major et al., 1993a). However, the final decay of transients would still be more rapid than postwashout. Whatever the distribution of sag conductances, their activation and deactivation would further "sculpt" PSPs.

Another factor to consider is the inward rectification shown by CA3 pyramidal cells: $R_{v}$ and $\tau_{0}$ are reduced at hyperpolarized potentials (Spruston and Johnston, 1992), even after washout of all the sag at the resting membrane potential (not shown). CA3 pyramidal cells recorded with sharp electrodes also demonstrate inward rectification (Brown et al., 1981; G. Major, unpublished observations). If $R_{m}$ is reduced by hyperpolarization, and behavior near any one hyperpolarized potential is effectively passive (as seems to be the case after washout), then there will be no single electrical geometry that describes the steady-state properties of the cell. However, at most potentials the basal and oblique dendrites will remain electrically compact. For a given input current, the early parts of the responses at hyperpolarized potentials will be similar to those at rest, since they are relatively insensitive to $R_{m}$ and $g_{\text {shun }}$ (e.g., Jonas et al., 1993; Major, 1993; Major ct al., 1993a,b).

\section{Other pyramidal cells modeled by the authors}

The range of specific electrical parameters found above is comparable to those in several other recent studies we have carried out using biocytin- or HRP-filled pyramidal neurons. In rat layer II/III visual cortical pyramidal cells, recorded with sharp electrodes, $C_{m}$ was about $0.65-0.8 \mu \mathrm{Fcm}^{-2}$, and $R_{i}$ was about 200 $300 \Omega \mathrm{cm}$ (Major, 1992). In these cells there were shunts of around 3-15 nS. Resting $R_{m}$ was around $50 \mathrm{k} \Omega \mathrm{cm}^{2}$, although there was considerable uncertainty about its exact value because it was ill-conditioned with respect to the size of the shunt. In one impaled adult rat hippocampal CAl pyramidal cell, $R_{i}$ was approximately $200 \Omega \mathrm{cm}$ (Major et al., 1993a). A mean $C_{m}$ of $0.85 \mu \mathrm{Fcm}^{-2}$ and a mean $R_{i}$ of about $370 \Omega \mathrm{cm}$ has been found recently in some preliminary models of three whole-cell recorded 20-d-old rat CA1 pyramidal cells. Unpublished models of three classes of neocortical pyramidal cell recorded with sharp electrodes (following Stratford et al., 1989), with $C_{m}$ constrained to $0.7 \mu \mathrm{Fcm}^{-2}$, yielded mean $R_{i}$ values of between about 250 and $400 \Omega \mathrm{cm}$. The sharp electrode data suffer from two main drawbacks compared with the whole-cell data: (1) the likely presence of a somatic shunt or, equivalently, a lower $R_{m}$ in the soma than in the dendrites, and (2) longer uncompensatable electrode artifacts (due largely to noncapacitative effects). However, the cells were from adult rats, which are more difficult to record from using whole-cell pipettes.

\section{Future experiments}

Given the importance of $R_{i}$ as a fundamental electrical parameter in neurons, experiments to measure it directly would yield data of great interest. Double impalcments of single cells (e.g., Wong and Stewart, 1992) or double whole-cell recordings (Stuart and Sakmann, 1994) might be useful when combined with accurate morphology. Reciprocity (if the two recording sites are A and $\mathrm{B}$, the A-to-B transfer response should equal the B-to-A response) could be added to linear scaling and absence of sag as a test for passive behavior. A complementary approach would be the use of voltage-sensitive dyes combined with cable modeling (Fromherz and Vetter, 1992), or dyes giving absolute or ratiometric estimates of membrane potential (e.g., Loew, 1993).

\section{Overview}

This study clearly demonstrates the potential of whole-cell recording combined with biocytin morphology as the basis for quantitative passive cable modeling of neurons. A general technique for fit rejection has been used to find confidence intervals for the electrical parameters of the models. Care has been taken to minimize systematic errors, but some problems remain. In the steady state, CA3 pyramidal cells have electrically compact dendritic trees, but identical synaptic inputs at different locations can generate PSPs of different shapes; there will also be serious voltage-clamp errors for fast events. The results of this study therefore have significant implications for the experimental analysis of synaptic inputs, and also suggest that real neurons have the potential for more computational power than the "point" units used in most neural network models.

\section{References}

Andersen P (1990) Synaptic integration in hippocampal CA1 pyramids. In: Progress in brain research, Vol 83, The hippocampus as a model system (Storm-Mathisen J, Zimmer J, Ottersen OP, eds), pp 215-222. New York: Elsevier.

Banks MI, Pearce RA, Smith PH (1993) Hyperpolarisation-activated cation current $\left(I_{t}\right)$ in neurons of the medial nucleus of the trapezoid body: voltage-clamp analysis and enhancement by norepincphrine and cAMP suggest a modulatory mechanism in the auditory brain stem. J Neurophysiol 70:1420-1432. 
Barrett JN, Crill WE (1974) Specific membrane properties of cat motoneurones. J Physiol (Lond) 239:301-324.

Beaulieu C, Colonnier M (1983) The number of neurones in the different laminae of the binocular and monocular regions of area 17 in the cat. J Comp Neurol 217:337-344.

Benz R, Frölich O, Läuger P, Montal M (1975) Electrical capacity of black lipid films and of lipid bilayers made from monolayers. Biochim Biophys Acta 394:323-334.

Bernander O, Douglas RJ, Martin K, Koch C (1991) Synaptic background activity determines spatio-temporal integration in single pyramidal cells. Proc Natl Acad Sci USA 88:1 1569-11573.

Bressloff PC (1993) Temporal processing in neural networks with adaptive short term memory: a compartmental model approach. Network 4:155-175.

Brown TH, Fricke RA, Perkel DH (1981) Passive electrical constants in three classes of hippocampal neurons. J Neurophysiol 46:812-827.

Butz EG, Cowan JD (1974) Transient potentials in dendritic systems of arbitrary geometry. Biophys J 14:661-689.

Clegg JS (1984a) Properties and metabolism of the aqueous cytoplasm and its boundaries. Am J Physiol 246:R133-R151.

Clegg JS (1984b) Intracellular water and the cytomatrix: some methods of study and current views. J Cell Biol 99:167s-171s.

Clements JD (1986) PhD thesis, Experimental Neurology Unit, John Curtin School of Medical Research, Australian National University, Canberra.

Clements JD, Redman SJ (1989) Cable properties of cat spinal motoneurons measured by combining voltage clamp, current clamp and intracellular staining. J Physiol (Lond) 409:63-87.

Colquhoun D, Jonas P, Sakmann B (1992) Action of brief pulses of glutamate on AMPA/kainate receptors in patches from different neurones of rat hippocampal slices. J Physiol (Lond) 458:261-287.

Connor JA, Ahmed Z (1984) Diffusion of ions and indicator dyes in neural cytoplasm. Cell Mol Neurobiol 4:53-66.

de Vries B, Principe JC (1992) The gamma model-a new neural model for temporal processing. Neural Networks 5:565-576.

Diggle PJ (1990) Time series: a biostatistical approach. London: Oxford UP.

Durand D, Carlen PL, Gurevich N, Ho A, Kunov H (1983) Electrotonic parameters of rat dentate granule cells measured using short current pulses and HRP staining. J Neurophysiol 50:1080-1097.

Edwards FA, Konnerth A, Sakmann B, Takahashi T (1989) A thin slice preparation for patch clamp recordings from neurones of the mammalian central nervous system. Pfluegers Arch 414:600-612.

Evans JD, Kember GC, Major G (1994) Techniques for the application of the analytical solution to the multi-cylinder somatic shunt cable model for passive neurones. Math Biosci, in press.

Fettiplace R, Andrews DM, Haydon DA (1971) The thickness, composition and structure of some lipid bilayers and natural membranes. J Membr Biol 5:277-296.

Fleshman JW, Segev I, Burke RE (1988) Electrotonic architecture of type-identified $\alpha$-motoneurons in the cat spinal cord. J Neurophysiol 60:60-85.

Fricke H (1931) The electrical conductivity and capacity of disperse systems. Physics 1:106-115.

Fromherz P, Vetter T (1992) Cable properties of arborized Retzius cells of the leech in culture as probed by a voltage-sensitive dye. Proc Natl Acad Sci USA 89:2041-2045.

Fulton AB (1982) How crowded is the cytoplasm? Cell 30:345-347.

Hama K, Arii T, Kosaka T (1989) Three-dimensional morphometric study of dendritic spines of the granule cell in the rat dentate gyrus with HVEM stereo images. J Electron Microsc Tech 6:175-183.

Hamill OP, Marty A, Neher E, Sakmann B, Sigworth FJ (1981) Improved patch-clamp techniques for high-resolution current recording from cells and cell-free membrane patches. Pfluegers Arch 391:85100.

Harris KM, Stevens JK (1989) Dendritic spines of CAl pyramidal cells in the rat hippocampus: serial electron microscopy with reference to their biophysical characteristics. J Neurosci 9:2982-2997.

Harris KM, Jensen FE, Stevens JK (1992) Three-dimensional structure of dendritic spines and synapses in rat hippocampus (CAl) at postnatal day 15 and adult ages: implications for the maturation of synaptic physiology and long-term potentiation. J Neurosci 12:26852705.

Haydon DA, Requena J, Urban BW (1980) Some effects of aliphatic hydrocarbons on the electrical capacity and ionic currents of the squid giant axon membrane. J Physiol (Lond) 309:229-245.
Hille B (1992) Ionic channels of excitable membranes, 2d ed. Sunderland, MA: Sinauer.

Hillman DE (1979) Neuronal shape parameters and substructures as a basis of neuronal form. In: The neurosciences: fourth study program (Schmitt FO, Worden FG, eds), pp 477-498. Cambridge, MA: MI I Press.

Hines M (1984) Efficient computation of branched nerve equations. Int J Biomed Comput 15:69-76.

Hines M (1989) A program for simulation of nerve equations with branching geometries. Int $\mathbf{J}$ Biomed Comput 24:55-68.

Holmes WR (1986a) Cable theory modeling of the effectiveness of synaptic inputs in cortical pyramidal cells, chapter 3. PhD thesis, University of California, Los Angeles.

Holmes WR (1986b) A continuous cable method for determining the transient potential in passive dendritic trees of known geometry. Biol Cybern 55:115-124.

Holmes WR, Rall W (1992) Estimating the electrotonic structure of neurons with compartmental models. J Neurophysiol 68:1438-1452.

Holmes WR, Segev I, Rall W (1992) Interpretation of time constant and electrotonic length estimates in multicylinder or branched neuronal structures. J Neurophysiol 68:1401-1420.

Horikawa K, Armstrong WE (1988) A versatile means of intracellular labeling: injection of biocytin and its detection with avidin conjugates. J Neurosci Methods 25:1-11.

Hounsgaard J, Midtgaard J (1989) Dendrite processing in more ways than one. Trends Neurosci 12:313-315.

Iansek R, Redman SJ (1973) An analysis of the cable properties of spinal motoneurones using a brief intracellular current pulse. J Physiol (Lond) 234:613-636.

Jack JJB, Miller S, Porter R, Redman SJ (1971) The time course of minimal excitatory post-synaptic potentials evoked in spinal motoneurones by group Ia afferent fibres. J Physiol (Lond) 215:353-380.

Jack JJB, Larkman AU, Major G, Mason AJR, Stratford KJ (1989) Simplified representations and compartmental modelling of cortical pyramidal neurones. J Physiol (Lond) 417:3P.

Jacobson K, Wojcieszyn J (1984) The translational mobility of substances within the cytoplasmic matrix. Proc Natl Acad Sci USA 81: 6747-6751.

Johnston D, Brown TH (1983) Interpretation of voltage-clamp measurements in hippocampal neurons. J Neurophysiol 50:464-486.

Jonas P, Major G, Sakmann B (1993) Quantal components of unitary EPSCs at the mossy fibre synapse on CA3 pyramidal cells of rat hippocampus. J Physiol (Lond) 472:615-663

Kushmeric MJ, Podolsky RJ (1969) Ionic mobility in muscle cells Science 166:1297-1298.

Lanczos C (1957) Applied analysis, pp 272-280. Englewood Cliffs, NJ: Prentice-Hall,

Landaw EM, DiStefano JJ III (1984) Multiexponential, multicompartmental, and noncompartmental modeling. II. Data analysis and statistical considerations. Am J Physiol 246:R665-R677.

Larkman AU (1991) Dendritic morphology of pyramidal neurones of the visual cortex of the rat: III. Spine distributions. J Comp Neurol 306:332-343.

Larkman A, Mason A (1990) Correlations between morphology and electrophysiology of pyramidal neurons in slices of rat visual cortex. I. Establishment of cell classes. J Neurosci 10:1407-1414.

Larkman A, Stratford K, Jack J (1991) Quantal analysis of excitatory synaptic action and depression in hippocampal slices. Nature 350 : 344-347.

Larkman AU, Major G, Stratford KJ, Jack JJR (1992) Dendritic morphology of pyramidal neurones of the visual cortex of the rat: IV. Electrical geometry. J Comp Neurol 323:153-166.

Loew LM (1993) Potentiometric membrane dyes. In: Fluorescent and luminescent probes for biological activity (Mason WT, ed), pp 150 160. London: Academic.

Major G (1992) The physiology, morphology and modelling of cortical pyramidal neurones. DPhil thesis, Laboratory of Physiology, Oxford University.

Major G (1993) Solutions for transients in arbitrarily branching cables: III. Voltage clamp problems. Biophys J 65:469-491.

Major G, Evans JD (1994) Solutions for transients in arbitrarily branching cables: IV. Non-uniform electrical parameters. Biophys J 66:615-634.

Major G, Larkman AU, Jack JJB (1990) Constraining non-uniqueness in passive electrical models of cortical pyramidal neurones. J Physiol (Lond) 430:23P. 
Major G, Evans JD, Jack JJB (1993a) Solutions for transients in arbitrarily branching cables: I. Voltage recording with a somatic shunt. Biophys J 65:423-449.

Major G, Evans JD, Jack JJB (1993b) Solutions for transients in arbitrarily branching cables: II. Voltage clamp theory. Biophys J 65 : $450-468$

Mastro AM, Babich MA, Taylor WD, Keith AD (1984) Diffusion of a small molecule in the cytoplasm of mammalian cells. Proc Natl Acad Sci USA 81:3414-3418.

Nelder JA, Mead R (1965) A geometric technique for optimisation. Comput J 7:308-327.

Nicoll A, Larkman A, Blakemore C (1993) Modulation of EPSP shape and efficacy by intrinsic membrane conductances in rat neocortical pyramidal neurons in vitro. J Physiol (Lond) 468:693-710.

Nitzan R, Segev I, Yarom Y (1990) Voltage behavior along the irregular dendritic structure of morphologically and physiologically characterized vagal motoneurons in the guinea pig. J Neurophysiol 63:333-346

Park MR, Kita H, Klee MR, Oomura Y (1983) Bridge balance in intracellular recording; introduction of the phase-sensitive method. $J$ Neurosci Methods 8:105-125.

Peters A, Kara DA, Harriman KM (1985) The neuronal composition of area 17 of rat visual cortex. III. Numerical considerations. J Comp Neurol 238:263-274

Pongracz F, Firestein S, Shepherd GM (1991) Electrotonic structure of olfactory sensory neurons analyzed by intracellular and whole cell patch techniques. J Neurophysiol 65:747-758.

Press WH, Flannery BP, Teukolsky SA, Vetterling WT (1988) Numerical recipes in C: the art of scientific computing. Cambridge: Cambridge UP.

Purves D (1981) Microelectrode methods for intracellular recording and ionophoresis. London: Academic.

Qian N, Sejnowski lJ (1989) An electro-diffusion model for computing membrane potentials and ionic concentrations in branching dendrites, spines and axons. Biol Cybern 62:1-15.

Rall W (1959) Branching dendritic trees and motoneuron membrane resistivity. Exp Neurol 1:491-527

Rall W (1964) Theoretical significance of dendritic trees for neuronal input-output relations. In: Neural theory and modelling (Reiss RF, ed), pp 73-97. Stanford, CA: Stanford UP.

Rall W (1967) Distinguishing theoretical synaptic potentials computed for different soma-dendritic distributions of synaptic input. J Neurophysiol 30:1138-1168.

Rall W (1969a) Time constants and electrotonic length of membrane cylinders and neurons. Biophys $\mathrm{J}$ 9:1483-1508.

Rall W (1969b) Distributions of potential in cylindrical coordinates and time constants for a membrane cylinder. Biophys J 9:1484-1 541 .

Rall W (1977) Core conductor theory and cable properties of neurons. In: Handbook of physiology. The nervous system. Cellular biology of neurones, Sect 1, Vol 1, Pt 1 (Kandel ER, ed), pp 39-98. Bethesda, MD: American Physiological Society.

Rall W, Segev I (1985) Space-clamp problems when voltage clamping branched neurons with intracellular microelectrodes. In: Voltage and patch clamping with microelectrodes (Smith TG Jr, Lecar H, Redman SJ, Gage P, eds), pp 191-215. Bethesda, MD: American Physiological Society.

Rall W, Burke RE, Smith TG, Nelson PG, Frank K (1967) Dendritic location of synapses and possible mechanisms for the monosynaptic EPSP in motoneurons. J Neurophysiol 30:1169-1193.

Rall W, Burke RE, Holmes WR, Jack JJB, Redman SJ, Segev I (1992) Matching dendritic neuron models to experimental data. Physiol Rev 72:S159-S186.

Rapp M, Yarom Y, Segev I (1992) The impact of parallel fiber background activity on the cable properties of cerebellar Purkinje cells. Neural Comput 4:518-533.

Rapp M, Segev I, Yarom Y (1994) Physiology, morphology and detailed passive models of cerebellar Purkinje cells. J Physiol (Lond) 474:101-118

Rinzel J, Rall W (1974) Transient response in a dendritic neuron model for current injected at one branch. Biophys J 14:759-790.

Rumelhart DE, McClelland JL (1986) Parallel distributed processing: explorations in the microstructure of cognition. Cambridge, MA: MIT Press.

Schanne O (1969) Measurements of cytoplasmic resistivity by means of the glass microelectrode. In: Glass microelectrodes (Levallee $\mathrm{M}$ Schanne OF, Hébert NC, eds), pp 299-321. New York: Wiley.

Schwan HP, Carstensen EL (1957) Dielectric properties of the membrane of lysed erythrocytes. Science 15:985-986.

Segev I, Fleshman JW, Miller JP, Bunow B (1985) Modeling the electrical properties of anatomically complex neurons using a network analysis program: passive membrane. Biol Cybern 53:27-40.

Shelton DP (1985) Membrane resistivity estimated for the Purkinje neuron by means of a passive computer model. Neuroscience 14:111131.

Spruston N, Johnston D (1992) Perforated patch-clamp analysis of the passive membrane properties of three classes of hippocampal neurons. J Neurophysiol 67:503-529.

Spruston N, Jaffe DB, Williams SH, Johnston D (1993) Voltage and space clamp errors associated with the measurement of electrotonically remote synaptic events. J Neurophysiol 70:78l-802.

Staley KJ, Otis TS, Mody I (1992) Membrane properties of dentate gyrus granule cells: comparison of sharp microelectrode and wholecell recordings. J Neurophysiol 67:1346-1358

Stevens JK, Trogadis J, Jacobs JR (1988) Development and control of axial neurite form: a serial electron microscopic analysis. In: Intrinsic determinants of neuronal form and function (Lasek RJ, Black MM, eds), pp 115-145. New York: Liss.

Stratford KJ, Mason AJR, Larkman AU, Major G, Jack JJB (1989) The modelling of pyramidal neurones in the visual cortex. In: The computing neurone (Durbin R, Miall C, Mitchison G, eds), pp 296321. Reading, England: Addison-Wesley.

Stuart GS, Sakmann B (1994) Active propagation of somatic action potentials into neocortical pyramidal cell dendrites. Nature 367:6973.

Takashima S (1976) Membrane capacity of squid giant axon during hyper- and depolarisations. J Membr Biol 27:21-39.

Takashima S, Schwan HP (1974) Passive electrical properties of squid axon membrane. J Membr Biol 17:51-68.

Tank DW, Hopfield JJ (1987) Neural computation by concentrating information in time. Proc Natl Acad Sci USA 84:1896-1900.

Thurbon D, Field A, Redman SJ (1994) Electrotonic profiles of interneurones in stratum pyramidale of the $C \wedge 1$ region of rat hippocampus. J Neurophysiol, in press.

Tokimasa T, Akasu T (1990) Cyclic AMP regulates an inward rectifying sodium-potassium current in dissocialed bull-frog sympathetic neurones. J Physiol (Lond) 420:409-429.

Turner DA (1984) Segmental cable evaluation of somatic transients in hippocampal neurons (CAI, CA3, and dentate). Biophys $\mathrm{J} 46: 73$ 84.

Turner DA (1988) Waveform and amplitude characteristics of evoked responses to dendritic stimulation of CAl guinea-pig pyramidal cells J Physiol (Lond) 395:419-439.

West MJ, Coleman PD, Flood DG (1988) Estimating the number of granule cells in the dentate gyrus with the disector. Brain Res 448 : $167-172$.

White JA, Manis PB, Young ED (1992) The parameter identification problem for the somatic shunt model. Biol Cybern 66:307-318.

Williams SH, Johnston D (1991) Kinetic properties of two anatomically distinct excitatory synapses in hippocampal CA3 pyramidal neurons. J Neurophysiol 66:1010-1020.

Wilson CJ (1987) Three-dimensional analysis of neuronal geometry using HVEM. J Electron Microsc Tech 6:175-183.

Wilson CJ, Park MR (1989) Capacitance compensation and bridge balance adjustment in intracellular recording from dendritic neurons. J Neurosci Methods 27:51-75.

Wojcieszyn JW, Schlegel RA, Wu E-S, Jacobson KA (1981) Diffusion of injected macromolecules within the cytoplasm of living cells. Proc Natl Acad Sci USA 78:4407-4410.

Wong RKS, Stewart M (1992) Different firing patterns generated in dendrites and somata of $\mathrm{CA} 1$ pyramidal neurones in guinea-pig hippocampus. J Physiol (Lond) 457:675-687. 